\title{
Neurobiologically Inspired Control of Engineered Flapping Flight
}

\author{
Soon-Jo Chung* and Michael Dorothy \\ University of Illinois at Urbana-Champaign, Urbana, Illinois 61801
}

DOI: $\underline{10.2514 / 1.45311}$

\begin{abstract}
This paper presents a new control approach and a dynamic model for engineered flapping flight with many interacting degrees of freedom. This paper explores the applications of neurobiologically inspired control systems in the form of central pattern generators to control flapping-flight dynamics. A rigorous mathematical and control theoretic framework to design complex three-dimensional wing motions is presented based on phase synchronization of nonlinear oscillators. In particular, we show that flapping-flying dynamics without a tail or traditional aerodynamic control surfaces can be effectively controlled by a reduced set of central pattern generator parameters that generate phase-synchronized or symmetry-breaking oscillatory motions of two main wings. Furthermore, by using Hopf bifurcation, we show that tailless aircraft alternating between flapping and gliding can be effectively stabilized by smooth wing motions driven by the central pattern generator network. Results of numerical simulation with a full six-degree-of-freedom flight dynamic model validate the effectiveness of the proposed neurobiologically inspired control approach.
\end{abstract}

\section{Nomenclature}

A

$a_{i}$
$\mathbf{B}$

$C_{D}\left(\alpha_{w}\right)$

$C_{L}\left(\alpha_{w}\right)$

d

F

$\mathbf{F}_{g}$

$F_{w x}, F_{w z}$

$\mathbf{f}\left(\mathbf{x}_{i} ; \rho_{i}\right)$

G

$\mathbf{I}_{b}$

$\mathbf{I}_{k}$

$k$

$k_{r}$

$\mathbf{L}$

$\ell$

M

$m$

$n$

$R$

$\mathbf{R}\left(\Delta_{i j}\right)$ and the tail, $\left(B_{x}, B_{y}, B_{z}\right)^{T}$ $(0,0, m g)^{T}$ with radius $\rho_{i}$ transformation $\in \mathbb{R}^{2 n \times 2 n}$

$=$ identity matrix $\in \mathbb{R}^{k \times k}$

$$
\left(M_{x}, M_{y}, M_{z}\right)^{T}, \mathrm{Nm}
$$

$=$ mass of the aircraft, $\mathrm{kg}$ network
$=$ additional forces generated by the body (fuselage) and the tail, $\left(A_{x}, A_{y}, A_{z}\right)^{T}$

$=$ amplitude bias of the $i$ th Hopf oscillator

$=$ additional aerodynamic moments from the body

$=$ local drag coefficient of the blade element

$=$ local lift coefficient of the blade element

$=$ location of the stroke and wing frames with respect to the body frame, $\left(d_{x}, d_{y}, d_{z}\right)^{T}$

$=$ total aerodynamic forces from the wing in the body frame (left, right), $\left(F_{x}, F_{y}, F_{z}\right)^{T}$

$=$ gravitational force vector in the inertial frame,

$=$ total aerodynamic forces of each wing in the $x$ and $z$ directions of the wing frame

$=$ Hopf nonlinear equations in the vector form

$=$ original Laplacian matrix with rotational

$=$ inertia matrix $\in \mathbb{R}^{3 \times 3}, \mathrm{~kg} \mathrm{~m}^{2}$

$=$ coupling gain of the coupled Hopf oscillators

$=$ reduced frequency of the flapping wing

$=$ graph Laplacian matrix $\in \mathbb{R}^{2 n \times 2 n}$

$=$ contraction rate of the virtual nonlinear system

$=$ total aerodynamic moments from each wing,

$=$ Total number of Hopf oscillators in the CPG

$=$ wingspan of a single wing, $\mathrm{m}$

$=2 \times 2$ rotational transformation matrix

Presented as Paper 1929 at the AIAA Infotech at Aerospace and Unmanned Unlimited Conference and Exhibit, Seattle, WA., 6-9 April 2009; received 6 May 2009; revision received 3 November 2009; accepted for publication 9 November 2009. Copyright $@ 2009$ by the American Institute of Aeronautics and Astronautics, Inc. All rights reserved. Copies of this paper may be made for personal or internal use, on condition that the copier pay the $\$ 10.00$ percopy fee to the Copyright Clearance Center, Inc., 222 Rosewood Drive, Danvers, MA 01923; include the code 0731-5090/10 and $\$ 10.00$ in correspondence with the CCC.

*Assistant Professor of Aerospace Engineering; sjchung@illinois.edu. Senior Member AIAA.

†Ph.D. Student, Department of Aerospace Engineering; dorothy1@ illinois.edu. $r$

$\mathbf{q}_{b}$

$\mathbf{T}_{b e}$

$\mathbf{T}_{b s}, \mathbf{T}_{s}$

$V_{b}$

$\mathbf{V}_{b}$

$V_{r}$

$\mathbf{V}_{w}$

$\mathbf{x}_{b}$

$\mathbf{x}_{i}$

$\mathbf{x}_{s}$

$\mathbf{x}_{w}$

$\alpha_{w}(r, t)$

$\alpha_{x}, \alpha_{y}$

$\beta_{w}(r, t)$

$\Delta_{i j}$

$\boldsymbol{\Theta}_{s}$

$\lambda$

$\lambda_{\text {max }}(\cdot)$

$\lambda_{\text {min }}(\cdot)$

$\rho_{i}$

$\sigma$

$\phi_{w}, \psi_{w}, \theta_{w}$

$\boldsymbol{\Omega}_{b}$

$\omega$

Subscripts

$b, s, w$

$i$

right, left
$=$ wingspan coordinate value $r \in[0, R]$ of the wing blade element, $\mathrm{m}$

$=$ Euler angles of the vehicle body with respect to the inertial frame, $\left(\phi_{b}, \theta_{b}, \psi_{b}\right)^{T}$, rad

$=$ directional cosine matrix of Euler angles $\in \mathbb{R}^{3 \times 3}$

$=$ angular transformation matrix

$=$ matrix of orthonormal eigenvectors of $\mathbf{L}$ without the ones vector $\in \mathbb{R}^{2 n \times 2(n-1)}$

$=$ speed of the vehicle, without the relative wind, $\mathrm{m} / \mathrm{s}$

$=$ vehicle velocity vector in the body frame, $\left(V_{b x}, V_{b y}, V_{b z}\right)^{T}, \mathrm{~m} / \mathrm{s}$

$=$ local wind speed of the wing blade element, $\mathrm{m} / \mathrm{s}$

$=$ total wind velocity vector of the blade element, $\left(V_{w x}, V_{w y}, V_{w z}\right)^{T} \mathrm{~m} / \mathrm{s}$

$=$ vehicle body-frame coordinates, $\left(x_{b}, y_{b}, z_{b}\right)$

$=$ state vector of the $i$ th Hopf oscillator, $\left(u_{i}, v_{i}\right)^{T}$

$=$ stroke-plane frame coordinates, $\left(x_{s}, y_{s}, z_{s}\right)$

$=$ wing frame coordinates (left, right), $\left(x_{w}, y_{w}, z_{s}\right)$

$=$ local angle of attack of the wing blade element, rad

$=$ angle of attack and slide-slip angle of the vehicle body, $\mathrm{rad} / \mathrm{s}$

$=$ local direction of the wind in the wing frame, rad

$=$ phase lead of the $i$ th Hopf oscillator from the $j$ th

$=$ stroke-plane inclination angle from the vertical line

$=$ common rate of convergence of Hopf oscillators

$=$ maximum eigenvalues of the matrix

$=$ minimum eigenvalues of the matrix

$=$ radius of the limit cycle from the $i$ th Hopf oscillator

$=$ bifurcation parameter $(1$ for a stable limit cycle or -1 for convergence to $a_{i}$ )

$=$ flapping, lead-lag, and pitch angles of each wing (left, right)

$=$ body angular rates, $(p, q, r)^{T}$, rad $/ \mathrm{s}$

$=$ common oscillation frequency of Hopf oscillators, $\mathrm{rad} / \mathrm{s}$ 


\section{Introduction}

$\mathbf{E}$ NGINEERED flapping flight holds promise for creating biomimetic micro aerial vehicles (MAVs) flying in lowReynolds-number regimes $\left(\operatorname{Re}<10^{5}\right)$, in which rigid fixed wings drop substantially in aerodynamic performance. MAVs are typically classified as having maximum dimensions of $15 \mathrm{~cm}$ and flying at a nominal speed of $1-20 \mathrm{~m} / \mathrm{s}$ in tight urban environments [1,2]. Although natural flyers such as bats, birds, and insects have captured the imaginations of scientists and engineers for centuries, the maneuvering characteristics of unmanned aerial vehicles are nowhere near the agility and efficiency of animal flight [3-5]. Such highly maneuverable MAVs will make paradigm-shifting advances in monitoring of critical infrastructures such as power grids, bridges, and borders, as well as in intelligence, surveillance, and reconnaissance applications.

The objective of this paper is to investigate whether the control and synchronization of coupled nonlinear oscillators, inspired by central pattern generators (CPGs) found in animal spinal cords, can control biomimetic flapping flight (see Fig. 1). An engineered CPG network, which ensures the stability and robust adaptation of motion, can significantly reduce the complexity associated with flapping flight. Unique to this research approach is the potential to reverse-engineer the key mechanisms of highly adaptive and robust rhythmic pattern modulations of flapping flight by integrating the neurobiological principles with the rigorous mathematical tools borrowed from nonlinear synchronization theory [6] and flight dynamics and controls. Such an approach has not been adopted for engineered flapping flight.

Although unsteady aerodynamics of flapping flight in lowReynolds-number regimes has been extensively studied through numerical $[\underline{2,7-17]}$ and experimental studies $[\underline{1}, \underline{18}-22]$, one of the most interesting and least understood aspects of flapping flight is how to precisely control and synchronize a large number of interacting limbs and joints that generate complex three-dimensional oscillatory movements of the wings governed by unsteady aerodynamic forces. There is relatively less prior work in control of flapping flight, with notable exceptions of $[19,23-26]$. In this paper, we focus on three stereotyped motion primitives to define the three-dimensional movements of wings: main flapping (stroke) motion (Fig. 2a), horizontal wing-sweeping motion called lead-lag motion (Fig. $\overline{2 b}$ ), and wing pitch twisting (Fig. 2c). Studying how to produce such synchronized wing motions is expected to shed light on the key characteristics of animal flapping flyers.

Previous robotic flapping flyers and their control design consider one or two degrees of freedom in the wings [17, 19,20,23, 24,27-34]. However, even insects such as the dragonfly (Anax parthenope) are reported to have complex three-dimensional movements by actively controlling flapping and twisting of four independent wings [3] .
Furthermore, prior studies in flapping flight $[\underline{1}-\underline{3}, \underline{5}, \underline{14}$ $16,18,22,33-38]$ assumed a very simple sinusoidal function for each joint to generate flapping oscillations, without deliberating on how multiple limbs (or their nervous systems) are connected and actuated to follow such a time-varying reference trajectory. However, as shall be seen later in this paper, the use of sinusoidal functions to generate oscillatory motions of the wings is not effective for stable and agile flapping-flying maneuvers, especially with time-varying oscillation frequency, amplitude, and bias. Experimental results using highspeed cameras have shown that the flapping motions in bats and birds are more complicated than perfect sinusoidal motions [3,21] with a fixed amplitude. Furthermore, we show in this paper that phase differences between multiple oscillators, which are not permitted with independent control of each wing joint, can be effective control mechanisms for flapping-flight dynamics. To bridge this gap, this paper aims to establish a novel adaptive CPG-based control theory for flapping flight through neuromechanical modeling, nonlinear control and synchronization, and numerical simulation.

The paper is organized as follows. We illustrate the fundamentals and advantages of the CPG-based control for engineered flapping flight in Sec. II. We present a mathematical and control theoretic formulation of synchronized motions of multiple joints in the wings and body in Sec. II.C. The kinematic modeling of three-dimensional multijoint wings and the dynamic models of flapping flight are presented in Sec. III. In Sec. IV, we present key control strategies and results of simulation with multijoint synchronization that enables a smooth transition between stable flapping flight and gliding flight. Concluding remarks are presented in Sec. V. We understand the challenges associated with building lightweight actuators that must be overcome to truly realize the potential of three-dimensional wing maneuvers. We present the fundamental neurobiologically inspired control theory that can further contribute to engineered flapping flight once such lightweight actuators become available in the future. In the meantime, we show how a multijoint robotic bat testbed (see Sec. III) driven by CPG control can further enhance our understanding of biomimetic flapping flight.

\section{Fundamentals of Neurobiologically Inspired Control}

This paper reports the first investigation of CPG models by using coupled limit-cycle oscillators for the purpose of controlled engineered flapping flight. We also introduce the use of Hopf bifurcation to enable smooth switching between flapping and gliding flight. Hooper [39] defines the central pattern generators of animals as neural networks that can endogenously (i.e., without rhythmic sensory or central brain input) produce coordinated patterns of rhythmic outputs. The self-sustained nature of CPGs is believed to

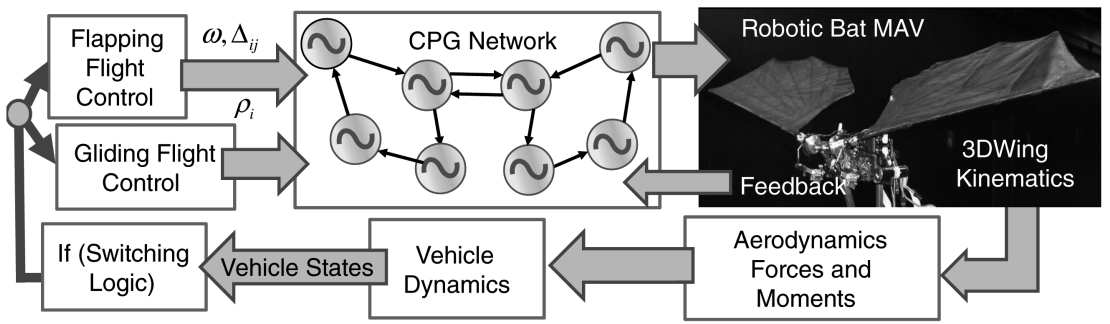

Fig. 1 Proposed hierarchical control structure with a CPG network with a reduced set of commands such as frequency $\omega(t)$, phase difference between wing-joint angles $\Delta_{i j}$, and maximum amplitude $\rho_{i}$.

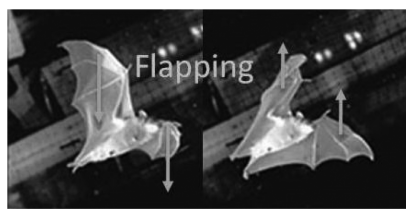

a) Flapping $\left(\phi_{w}\right)$

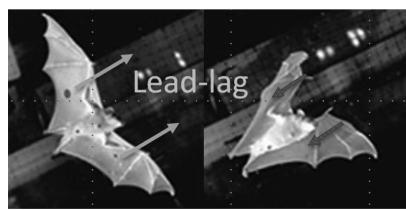

b) Lead-leg sweeping $\left(\psi_{w}\right)$

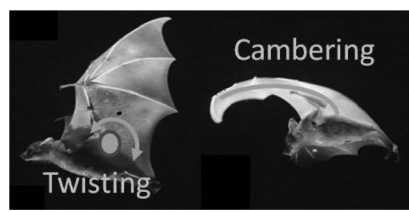

c) Pitch $\left(\theta_{w}\right)$ and cambering

Fig. 2 Basic wing movements of bats employed in this paper (images from [22]). Except for cambering, birds exhibit similar wing movements. 
reduce the computation burden of the brain. As illustrated in Fig. 1, the central controller, similar to the brain of an animal, is expected to stabilize the vehicle dynamics by commanding a reduced number of variables such as the frequency $\omega(t)$ and phase difference $\Delta_{i j}$ of the oscillators instead of directly controlling multiple joints. The existence of CPGs has been confirmed by biologists [39-47]. Experiments with limbed vertebrates have shown that individual limbs can produce rhythmic movements endogenously [39,48]. Such empirical data have been interpreted as evidence that each limb has its own CPGs that can behave in a self-sustained way. However, sensory feedback is also known to play a crucial role in altering motor patterns $[39,49]$ to cope with environmental perturbations. Incorporation of sensory feedback into the CPG model has been presented in companion papers $[50,51]$ for a turtle robot.

The most popular animal model for CPGs has been the lamprey, a primitive eel-like fish [52]. Although the robotics community eagerly embraced the concept of CPG models for swimming or walking robots [50,53-55], this work reports the first CPG-based control for flapping flight. The use of nonlinear oscillators for insect flapping flight has also been suggested by some biologists [37,38]. Clearly, flapping flight is technically more challenging to mimic than swimming and walking, due to its uncompromising aerodynamic characteristics.

\section{A. Mathematical Model of CPGs by Hopf Oscillators}

Our neurobiologically inspired approach centers on deriving an effective mathematical model of CPGs based on coupled nonlinear limit-cycle dynamics. Once neurons form reciprocally inhibiting relations, they oscillate and spike periodically. Some prior work uses a discrete nonlinear equation that describes spiking and spikingbursting of a neuron model [53]. In the present paper, an abstract mathematical model of complicated neuron models is obtained by coupled nonlinear limit cycles that essentially exhibit the rhythmic behaviors of coupled neuronal networks. In the field of nonlinear dynamics, a limit cycle is defined as an isolated closed trajectory that exhibits self-sustained oscillation $[56,57]$. If stable, small perturbations (initial conditions) will be forgotten and the trajectories will converge to the limit cycle. This superior robustness makes a limit cycle an ideal simplified dynamic model of CPGs.

In the present paper, we use the following limit-cycle model called the Hopf oscillator, named after the supercritical Hopf bifurcation model with $\sigma=1$ :

$$
\begin{aligned}
& \frac{\mathrm{d}}{\mathrm{d} t}\left(\begin{array}{c}
u-a \\
v
\end{array}\right)=\left[\begin{array}{cc}
-\lambda\left(\frac{(u-a)^{2}+v^{2}}{\rho^{2}}-\sigma\right) & -\omega(t) \\
\omega(t) & -\lambda\left(\frac{(u-a)^{2}+v^{2}}{\rho^{2}}-\sigma\right)
\end{array}\right] \\
& \quad \times\left(\begin{array}{c}
u-a \\
v
\end{array}\right)+\mathbf{u}(t)
\end{aligned}
$$

Equivalently,

$$
\dot{\mathbf{x}}=\mathbf{f}(\mathbf{x} ; \rho ; \sigma)+\mathbf{u}(t), \quad \text { with } \quad \mathbf{x}=(u-a, v)^{T}
$$

where the limiting cycle of the Hopf oscillator is a circle with a radius $\rho>0$, for which the center is at $(a, 0)^{T}, \lambda>0$ denotes the convergence rate to the circular limit cycle, and $\mathbf{u}(t)$ is an external or coupling input. For a single Hopf oscillator with $\mathbf{u}(t)=0$, a Lyapunov function

$$
V=\left(\frac{(u-a)^{2}+v^{2}}{\rho^{2}}-1\right)^{2}
$$

can be used to prove global asymptotic stability to the circular limit cycle. The bifurcation parameter $\sigma$ can switch from 1 to -1 such that

$$
\left(\frac{(u-a)^{2}+v^{2}}{\rho^{2}}+1\right)
$$

This would change the stable limit-cycle dynamics to the dynamics with a globally stable equilibrium point at the bias $a$ (see [56]). Such a change can be used to turn the flapping oscillatory motion to the gliding mode, as shall be seen in Sec. IV. We assume $\sigma=1$ unless noted otherwise. For coupled Hopf oscillators, the stability proof is much more involved and discussed in Sec. II.C.

The possibly time-varying parameter $\overline{\omega(t)}>0$ determines the oscillation frequency of the limit cycle. A time-varying $a(t)$ sets the bias to the limit cycle such that it converges to $u(t)=\rho \cos (\omega t+$ $\delta)+a$ and $v(t)=\rho \sin (\omega t+\delta)$ on a circle. This bias $a$ does not change the results of the stability proof. The output variable to generate the desired oscillatory motion of each joint is the first state $u$ from the Hopf oscillator model in Eq. (1).

There are two specific reasons why we prefer the Hopf oscillator to construct engineered CPG arrays (e.g., see the salamander robot $[54,58]$ and the turtle robot $[50,51])$. One essential property of the Hopf oscillator for our synchronization stability proof is that its limit cycle is a symmetric circle, as opposed to Van der Pol oscillators [40] or Rayleigh oscillators [56]. Hence, we can verify the following:

$$
\begin{gathered}
\mathbf{f}(\mathbf{R}(\Delta) \mathbf{x} ; \rho ; \sigma)=\mathbf{R}(\Delta) \mathbf{f}(\mathbf{x} ; \rho ; \sigma) \\
\mathbf{R}(\Delta)=\left[\begin{array}{cc}
\cos \Delta & -\sin \Delta \\
\sin \Delta & \cos \Delta
\end{array}\right]
\end{gathered}
$$

where $\mathbf{R}(\Delta) \in \mathcal{S O}(2)$ is a 2-D rotational transformation such that $\mathbf{R}(-\Delta)=\mathbf{R}^{-1}(\Delta)=\mathbf{R}^{T}(\Delta)$. Additionally, this symmetry prevents the classical problem of instability due to switching between two stable systems, as one of the systems would need to be asymmetric [59]. The stability of coupled Hopf oscillators has also been investigated in $[4,51,60]$.

Second, our stability proof of phase synchronization exploits a scaling factor of a symmetric oscillator

$$
\mathbf{f}(g \mathbf{x} ; \rho ; \sigma)=g \mathbf{f}(\mathbf{x} ; \rho / g ; \sigma)
$$

\section{B. Rationales for CPG-Based Flapping Flight Control}

We articulate the three key advantages of CPG-based control for control of a flapping-flying vehicle with complex wing kinematics: 1) reduced dimensionality, 2) adaptive pattern modulation, and 3) synchronization.

\section{Reduced Dimensionality and Bandwidth Requirement}

The CPGs in animal spinal cords are known to relieve the computation burden of locomotion in the brain [39,52]. Similarly, one significant advantage of CPG-based control over conventional control approaches is that CPG-based control reduces the dimensionality and bandwidth of signals required from the main controller to its actuators. As shown in Fig. 1, the main outer-loop flight controller needs to command only the reduced number of CPG parameters (e.g., frequency, phase lag, and amplitude) and much less frequently, instead of directly commanding time-specific reference signals for all the degrees of freedom in the wings and the body. Hence, the use of engineered CPGs can be regarded as implementing motion primitives [61] for systems with many interacting degrees of freedom. For example, a learning-based controller [62] using CPGs needs to adapt only the reduced-dimensionality $\overline{\mathrm{CPG}}$ parameters. Such a model reduction approach for flight control has not been exploited in the literature. The reduced dimensionality of the CPGbased approach makes learning-based adaptive flight control more practical.

\section{Adaptive Pattern Modulation}

Birds and bats modulate the CPG parameters (frequency, phase difference, bias, and amplitude) for the flapping, twisting, lead-lag, cambering, and flexing of the wings during their flight as a function of flight speed $[3,63,64]$ and flight modes (e.g., turning, gliding, soaring, cruising, hovering, and perching). High-speed film analyses $[\underline{3}, \underline{63}]$ reveal that the flapping angle and frequency are largest at zero forward speed or in hovering flight, and they decrease with increasing flight speed $V$ (e.g., $\propto V^{-0.277}$ for some bats [63]). Such 
time-varying CPG parameters, shown in Fig. 1, will change the shape, size, and flexing of the wings, which constitute the morphological flight parameters [5]. Prior studies in flapping flight, although true in steady flight, assume that there is a constant or very narrow range of optimal frequency or amplitude $[\underline{1}, \underline{2}, \underline{8}-$ $16,19,23,32,34]$. Agile vehicles with multiple flight modes may require a large envelope or discontinuous parameter changes. Typical sinusoidal signals can be modulated as well for continuous and discontinuous changes in frequency by using an integral of frequency, but discontinuous changes in amplitude or bias would require a low-pass filter, thereby adding phase delays. The Hopf oscillator guarantees continuous transitions for any time-varying input of these parameters, without a need for any low-pass filtering. This same behavior extends to the ability to handle any initial conditions and the ability to reject disturbances.

\section{Synchronization of Symmetric and Symmetry-Breaking Oscillation}

Bats exhibit complex wing flapping motions generated by their multijointed and compliant wings. One aim of the neurobiological approach to engineered flapping flight is to produce the analytical model of a wing beat oscillator that matches empirical data $[21,22,63,65]$. While the benefits of nonlinear limit cycles for CPG models are articulated above, deriving an effective CPG model for engineered flapping flight has been largely an open problem (e.g., limit-cycle dynamics, network topology, and how to integrate input and feedback signals). The key research issues include how to ensure the amplitude or phase synchronization of multiple coupled CPG oscillators and how to opportunistically break the symmetry of the oscillators for performing agile maneuvering of a small flappingflight vehicle. Unique to this formulation of coupled oscillators is the ability to set and control the phase differences between multiple oscillators (see $\Delta_{i j}$ in Fig. 1). In contrast, the use of an independently controlled sinusoidal function for each wing joint would not permit a control law that uses a phase difference $\Delta_{i j}$. Furthermore, stability proofs of coupled sine functions are largely open problems and more difficult to handle than the standard form of limit-cycle oscillators such as $\dot{\mathbf{x}}=\mathbf{f}(\mathbf{x})+\mathbf{u}(t)$. Observations of birds have found these phase differences to be key in performing maneuvers [66], and we will later see that they can be useful for vehicle stability. In the next section, we present how to construct stable coupled Hopf oscillators for the purpose of adaptive pattern modulation.

\section{Global Exponential Synchronization of CPG Oscillators}

Synchronization means an exact match of the scaled amplitude with a desired phase difference in this paper. Hence, phase synchronization permits different actuators to oscillate at the same frequency but with a prescribed phase lead or lag. However, a sinusoidal function is not adequate to entail the complex coupling and synchronization between various joints and limbs. Hence, the use of coupled nonlinear oscillators in this paper provides a feasible solution to construct complex synchronized motions of multiple wing joints. In essence, each CPG dynamic model in Eq. (1) is responsible for generating the limiting oscillatory behavior of a corresponding joint, and the diffusive coupling among CPGs reinforces phase synchronization. For example, the flapping angle has roughly a $90 \mathrm{deg}$ phase difference with the pitching joint to maintain the positive angle of attack (see the actual data from birds in [3]). The oscillators are connected through diffusive couplings, and the $i$ th
Hopf oscillator can be rewritten with a diffusive coupling with the phase-rotated neighbor:

$$
\dot{\mathbf{x}}_{i}=\mathbf{f}\left(\mathbf{x}_{i} ; \rho_{i}\right)-k(t) \sum_{j \in \mathcal{N}_{i}}^{m_{i}}\left(\mathbf{x}_{i}-\frac{\rho_{i}}{\rho_{j}} \mathbf{R}\left(\Delta_{i j}\right) \mathbf{x}_{j}\right)
$$

where the Hopf oscillator dynamics $\mathbf{f}\left(\mathbf{x}_{i} ; \rho_{i}\right)$ with $\sigma=1$ is defined in Eq. (1), $\mathcal{N}_{i}$ denotes the set that contains only the local neighbors of the $i$ th Hopf oscillator, and $m_{i}$ is the number of the neighbors. The $2 \times 2$ matrix $\mathbf{R}\left(\Delta_{i j}\right)$ is a 2-D rotational transformation of the phase difference $\Delta_{i j}$ between the $i$ th and $j$ th oscillators. The positive (or negative) $\Delta_{i j}$ indicates how much phase the $i$ th member leads (or lags) from the $j$ th member and $\Delta_{i j}=-\Delta_{j i}$. The positive scalar $k(t)$ denotes the coupling gain and can be time-varying for different flight modes.

We construct as many degrees of freedom as needed to more accurately model the joints of the wings, but let us focus on the key three flapping motions defined in Fig. 2: namely, flapping angle $\phi_{w}$, wing pitch (twisting) angle $\theta_{w}$, and wing lead-lag angle $\psi_{w}$. Additionally, we assume that there is a second flapping joint $\phi_{w 2}$ in the wing that can reduce the drag in the upstroke by folding the wings toward the body. Then we can construct the whole state vector of the coupled oscillator such as

$$
\{\mathbf{x}\}=\left(\begin{array}{c}
\mathbf{x}_{1} \\
\mathbf{x}_{2} \\
\mathbf{x}_{3} \\
\mathbf{x}_{4} \\
\mathbf{x}_{5} \\
\mathbf{x}_{6} \\
\mathbf{x}_{7} \\
\mathbf{x}_{8}
\end{array}\right)=\left(\begin{array}{c}
\left(u_{1}-a_{1}, v_{1}\right)^{T} \\
\left(u_{2}-a_{2}, v_{2}\right)^{T} \\
\left(u_{3}-a_{3}, v_{3}\right)^{T} \\
\left(u_{4}-a_{4}, v_{4}\right)^{T} \\
\left(u_{5}-a_{5}, v_{5}\right)^{T} \\
\left(u_{6}-a_{6}, v_{6}\right)^{T} \\
\left(u_{7}-a_{7}, v_{7}\right)^{T} \\
\left(u_{8}-a_{8}, v_{8}\right)^{T}
\end{array}\right)=\left(\begin{array}{c}
\left(\phi_{w_{R}}-a_{1}, v_{1}\right)^{T} \\
\left(\theta_{w_{R}}-a_{2}, v_{2}\right)^{T} \\
\left(\psi_{w_{R}}-a_{3}, v_{3}\right)^{T} \\
\left(\phi_{w 2_{R}}-a_{4}, v_{4}\right)^{T} \\
\left(\phi_{w_{L}}-a_{5}, v_{5}\right)^{T} \\
\left(\theta_{w_{L}}-a_{6}, v_{6}\right)^{T} \\
\left(\psi_{w_{L}}-a_{7}, v_{7}\right)^{T} \\
\left(\phi_{w 2_{L}}-a_{8}, v_{8}\right)^{T}
\end{array}\right)
$$

Note that $\mathbf{x}_{i}$ here might represent the shifted Hopf oscillator vector such that $\mathbf{x}_{i}=\left(u_{i}-a_{i}, v_{i}\right)^{T}$, as shown in Eq. (1), where $a_{i}(t)$ is the center of oscillation. For example, if we need a $10 \mathrm{deg}$ offset for the main flapping stroke angle $\phi_{w}$, then we can set $a_{1}=a_{5}=10 \mathrm{deg}$ so that the flapping stroke angle oscillates around $10 \mathrm{deg}$.

To analyze the stability of a network, we need to construct fully coupled dynamics of the augmented state vector $\{\mathbf{x}\}$ from Eq. (4):

$$
\{\dot{\mathbf{x}}\}=[\mathbf{f}(\{\mathbf{x}\} ; \rho)]-k(t) \mathbf{G}\{\mathbf{x}\}
$$

where

$$
[\mathbf{f}(\{\mathbf{x}\} ; \rho)]=\left[\mathbf{f}\left(\mathbf{x}_{1} ; \rho_{1}\right) ; \mathbf{f}\left(\mathbf{x}_{2} ; \rho_{2}\right) ; \cdots ; \mathbf{f}\left(\mathbf{x}_{n} ; \rho_{n}\right)\right]
$$

and $\rho=\left(\rho_{1}, \ldots, \rho_{n}\right)^{T} \in \mathbb{R}^{n}$, implying that the radius of each oscillator can be different. The $2 n \times 2 n$ matrix $\mathbf{G}$ is a Laplacian matrix, with phase shifts $\mathbf{R}\left(\Delta_{i j}\right)$ constructed from Eq. (4).

The coupling topology and phase shift between each oscillators are reflected in the $\mathbf{G}$ matrix. Such phase shifts along with the bifurcation parameter $\sigma$ can be used to define different flight modes, similar to walking gaits. Numerous configurations are possible as long as they are on balanced graphs [6] and we can choose either a bidirectional or a unidirectional coupling between the oscillators. Some configurations considered in this paper are shown in Fig. 3. We can choose either a bidirectional (e.g., configuration B) or unidirectional (e.g.,

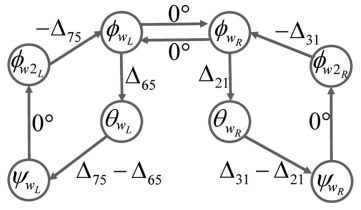

a)

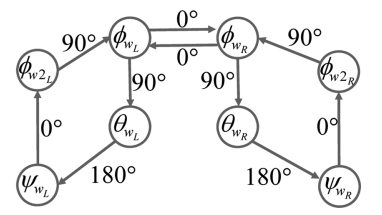

b)

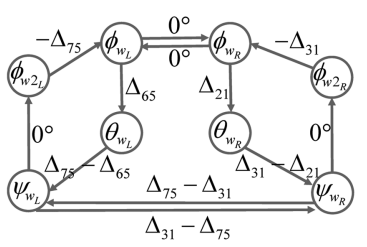

c)

Fig. 3 Graph configurations of the coupled Hopf oscillators on balanced graphs: a) configuration A, b) nominal symmetric configuration A, and c) configuration $\mathbf{B}$. 
configuration A) coupling between the oscillators. The numbers next to the arrows indicate the phase shift $\Delta_{i j}$; hence, $\Delta_{i j}>0$ indicates how much phase the $i$ th member leads. Since the graphs in Fig. 3 are on balanced graphs, the number of input ports equal the number of output ports. Further, all the phase shifts $\Delta_{i j}$ along one cycle should add up to a modulo of $2 \pi$. Figure $3 \mathrm{~b}$ shows the nominal values of the phase shift from the symmetric wing configuration such that $\Delta_{21}=$ $\Delta_{65}=90 \mathrm{deg}$ and $\Delta_{31}=\Delta_{75}=-90 \mathrm{deg}$. The empirical data suggest that the pitching angle $\theta_{w}$ has approximately a 90 deg phase lag with the flapping angle $\phi_{w}$, which agrees with the aerodynamically optimal value $[3,20]$. For hovering flight, Dickinson et al. [20], using their Robofly testbed and numerical simulations, found that increasing the phase-difference value $\Delta_{21}$ to $90 \mathrm{deg}+\delta$ further contributed to enhancing the lift generation, which is explained by the wake capture and rotational circulation lift mechanism. Hence, the ability to control $\Delta_{21}$ allows us to investigate the optimal value of the phase difference. In addition, the nominal value of $\Delta_{31}=-90 \mathrm{deg}$, the phase difference between the flapping stroke angle and lead-lag angle, will result in an elliptical orbit of the wing. On the other hand, by having two difference phase differences for the left and right wings, we can investigate how symmetric-breaking wing rotations contribute to the agile turning of flapping flight. Furthermore, by having an independent control of the phase differences $\Delta_{31}$ and $\Delta_{75}$, we can investigate another symmetry-breaking impact of the differential delay in the lead-lag motion. Such differential phases can be used to stabilize the flapping-flying dynamics.

The $\mathbf{G}$ matrix in Eq. () for Fig. $\underline{3 a}$ can be found as

$\begin{array}{cc}\mathbf{0} & \mathbf{0} \\ \mathbf{I}_{2} & \mathbf{0} \\ -\frac{\rho_{3}}{\rho_{2}} \mathbf{R}\left(\Delta_{31}-\Delta_{21}\right) & \mathbf{I}_{2} \\ \mathbf{0} & -\frac{\rho_{4}}{\rho_{3}} \mathbf{I}_{2} \\ \mathbf{0} & \mathbf{0} \\ \mathbf{0} & \mathbf{0} \\ \mathbf{0} & \mathbf{0} \\ \mathbf{0} & \mathbf{0}\end{array}$

$\frac{\rho_{1}}{\rho_{4}} \mathbf{R}\left(\Delta_{31}\right)$
$\mathbf{0}$
$\mathbf{0}$
$\mathbf{I}_{2}$
$\mathbf{0}$
$\mathbf{0}$
$\mathbf{0}$
$\mathbf{0}$

Proof 1: The proof is given in [67] by computing $(\mathrm{d} / \mathrm{d} t) \delta \mathbf{z}^{T} \delta \mathbf{z}=2 \delta \mathbf{z}^{T} \mathbf{F} \delta \mathbf{z}$.

The synchronized flow-invariant subspace for the configuration in Fig. $\underline{3 a}$ is defined by $\mathbf{G}\{\mathbf{x}\}=\mathbf{0}$ such that

$$
\begin{gathered}
\mathcal{M}(\{\mathbf{x}\}) \Longleftrightarrow \mathbf{x}_{1}=\frac{\rho_{1}}{\rho_{2}} \mathbf{R}\left(\Delta_{12}\right) \mathbf{x}_{2}=\frac{\rho_{1}}{\rho_{3}} \mathbf{R}\left(\Delta_{13}\right) \mathbf{x}_{3}=\frac{\rho_{1}}{\rho_{4}} \mathbf{R}\left(\Delta_{13}\right) \mathbf{x}_{4} \\
=\frac{\rho_{1}}{\rho_{5}} \mathbf{x}_{5}=\frac{\rho_{1}}{\rho_{6}} \mathbf{R}\left(\Delta_{56}\right) \mathbf{x}_{6}=\frac{\rho_{1}}{\rho_{7}} \mathbf{R}\left(\Delta_{57}\right) \mathbf{x}_{7}=\frac{\rho_{1}}{\rho_{8}} \mathbf{R}\left(\Delta_{57}\right) \mathbf{x}_{8}
\end{gathered}
$$

where we used $\Delta_{i j}=-\Delta_{j i}$.

The flow-invariant subspace $\mathcal{M}$ in Eq. (9) can be rewritten with respect to the first state vector $\mathbf{x}_{1}=\mathbf{z}_{1}$ such that

$$
\mathcal{M}(\{\mathbf{x}\}) \Longleftrightarrow \mathbf{z}_{1}=\mathbf{z}_{2}=\cdots=\mathbf{z}_{n}, \quad\{\mathbf{z}\}=\mathbf{T}\left(\Delta_{i j}, \rho_{i}\right)\{\mathbf{x}\}
$$

where

$$
\begin{gathered}
\{\mathbf{z}\}=\left(\mathbf{z}_{1}, \mathbf{z}_{2}, \ldots, \mathbf{z}_{\mathbf{n}}\right)^{\mathbf{T}}, \quad \mathbf{z}_{1}=\mathbf{x}_{1}, \quad \mathbf{z}_{2}=\frac{\rho_{1}}{\rho_{2}} \mathbf{R}\left(\Delta_{12}\right) \mathbf{x}_{2} \\
\mathbf{z}_{3}=\frac{\rho_{1}}{\rho_{3}} \mathbf{R}\left(\Delta_{13}\right) \mathbf{x}_{3}
\end{gathered}
$$

and so on. For example, the T matrix for the configuration in Fig. 3a is given as where, in general, the radii (the amplitude of the oscillation from the bias $a_{i}$ ) are symmetric such that $\rho_{1}=\rho_{2}, \rho_{2}=\rho_{6}, \rho_{4}=\rho_{7}$, and $\rho_{5}=\rho_{8}$, although the difference of the maximum amplitude of each oscillation can occasionally be used to generate side forces or turning (rolling or yawing) moments.

The proof of phase synchronization boils down to finding the condition of $k$ by which the flow-invariant synchronized state [60], constructed from $\mathbf{G}\{\mathbf{x}\}=\mathbf{0}$, is globally stable. In fact, by using contraction theory $[\underline{60}, 67]$, we can prove global exponential synchronization of the coupled Hopf oscillators. We first introduce the main theorem of contraction theory.

Theorem 1: For the system $\dot{\mathbf{x}}=\mathbf{f}(\mathbf{x}, t)$, if there exists a uniformly positive definite metric

$$
\mathbf{M}(\mathbf{x}, t)=\boldsymbol{\Theta}(\mathbf{x}, t)^{T} \boldsymbol{\Theta}(\mathbf{x}, t)
$$

where $\boldsymbol{\Theta}$ is some smooth coordinate transformation of the virtual displacement $\delta \mathbf{z}=\boldsymbol{\Theta} \delta \mathbf{x}$, such that the associated generalized Jacobian $F$ is uniformly negative definite, i.e., $\exists \ell>0$ such that

$$
\mathbf{F}=\left(\dot{\boldsymbol{\Theta}}(\mathbf{x}, t)+\boldsymbol{\Theta}(\mathbf{x}, t) \frac{\partial \mathbf{f}}{\partial \mathbf{x}}\right) \boldsymbol{\Theta}(\mathbf{x}, t)^{-1} \leq-\ell \mathbf{I}
$$

Then all system trajectories converge globally to a single trajectory exponentially fast, regardless of the initial conditions, with a global exponential convergence rate of the largest eigenvalues of the symmetric part of $\mathbf{F}$. Such a system is said to be contracting.

$\left.\begin{array}{cccc}-\frac{\rho_{1}}{\rho_{5}} \mathbf{I}_{2} & \mathbf{0} & \mathbf{0} & \mathbf{0} \\ \mathbf{0} & \mathbf{0} & \mathbf{0} & \mathbf{0} \\ \mathbf{0} & \mathbf{0} & \mathbf{0} & \mathbf{0} \\ \mathbf{0} & \mathbf{0} & \mathbf{0} & \mathbf{0} \\ 2 \mathbf{I}_{2} & \mathbf{0} & \mathbf{0} & \frac{\rho_{5}}{\rho_{8}} \mathbf{R}\left(\Delta_{75}\right) \\ -\frac{\rho_{6}}{\rho_{5}} \mathbf{R}\left(\Delta_{65}\right) & \mathbf{I}_{2} & \mathbf{0} & \mathbf{0} \\ \mathbf{0} & -\frac{\rho_{7}}{\rho_{6}} \mathbf{R}\left(\Delta_{75}-\Delta_{65}\right) & \mathbf{I}_{2} & \mathbf{0} \\ \mathbf{0} & \mathbf{0} & -\frac{\rho_{8}}{\rho_{7}} \mathbf{I}_{2} & \mathbf{I}_{2}\end{array}\right]$

$$
\begin{gathered}
\mathbf{T}\left(\Delta_{i j}, \rho_{i}\right)=\operatorname{diag}\left(\mathbf{I}_{2}, \frac{\rho_{1}}{\rho_{2}} \mathbf{R}\left(\Delta_{12}\right), \frac{\rho_{1}}{\rho_{3}} \mathbf{R}\left(\Delta_{13}\right), \frac{\rho_{1}}{\rho_{4}} \mathbf{R}\left(\Delta_{13}\right), \frac{\rho_{1}}{\rho_{5}} \mathbf{I}_{2},\right. \\
\left.\frac{\rho_{1}}{\rho_{6}} \mathbf{R}\left(\Delta_{56}\right), \frac{\rho_{1}}{\rho_{7}} \mathbf{R}\left(\Delta_{57}\right), \frac{\rho_{1}}{\rho_{8}} \mathbf{R}\left(\Delta_{57}\right)\right)
\end{gathered}
$$

Then we present the main theorem of this section.

Theorem 2: If the following condition is met uniformly for $\forall t>0$, any initial condition $\{\mathbf{x}\}$ of the coupled Hopf oscillators in Eqs. (4) and (6) on a balanced graph converges to the flow-invariant synchronized state $\mathcal{M}$ exponentially fast:

$$
k(t) \lambda_{\min }\left(\mathbf{V}^{T}\left(\mathbf{L}+\mathbf{L}^{T}\right) \mathbf{V} / 2\right)>\lambda
$$

where $\lambda$ is the convergence rate of the Hopf oscillator in Eq. (1), $\lambda_{\min }\left(\mathbf{V}^{T}\left(\mathbf{L}+\mathbf{L}^{T}\right) \mathbf{V} / 2\right)$ denotes the minimum eigenvalue, and $\mathbf{L}$ is the Laplacian matrix constructed from the balanced graph such that $\mathbf{G}=\mathbf{T}^{-1} \mathbf{L} \mathbf{T}$ with $\mathbf{T}$ defined from Eq. (10). In addition, the real orthonormal $2 n \times 2(n-1)$ matrix $\mathbf{V}$ is constructed from the orthonormal eigenvectors of $\left(\mathbf{L}+\mathbf{L}^{T}\right) / 2$ other than the ones vector $\mathbf{1}=\left(\mathbf{I}_{2} ; \mathbf{I}_{2} ; \cdots ; \mathbf{I}_{2}\right)$ such that $\mathbf{V V}^{T}+\mathbf{1 1}^{T} / n=\mathbf{I}_{2 n}$.

Proof 2: The proof can be obtained by exploiting results from [60]. The proof here is simpler than $[50,51]$ in the sense that we derive the Laplacian matrix and orthonormal flow-invariant matrix that are independent of the rotational angles. Consider the orthonormal space $\mathbf{V}$, constructed from the orthonomal eigenvectors of the symmetric part of $\mathbf{L}$ (see [6]). Then the global exponential convergence to the flow-invariant synchronized state $\mathcal{M}$ is equivalent to 


$$
\mathbf{V}^{T}\{\mathbf{z}\} \rightarrow \mathbf{0}
$$

By premultiplying Eq. (6) by $\mathbf{T}$ and using $\mathbf{T}\{\mathbf{x}\}=\{\mathbf{z}\}$ and $\mathbf{G}=\mathbf{T}^{-1} \mathbf{L} \mathbf{T}$, we can obtain

$$
\{\dot{\mathbf{z}}\}=\mathbf{T}[\mathbf{f}(\{\mathbf{x}\} ; \rho)]-k(t) \mathbf{L}\{\mathbf{z}\}
$$

where the CPG network in the example in Fig. 3a is on a balanced graph such that

$$
\mathbf{L}=\left[\begin{array}{cccccccc}
2 \mathbf{I}_{2} & \mathbf{0} & \mathbf{0} & -\mathbf{I}_{2} & -\mathbf{I}_{2} & \mathbf{0} & \mathbf{0} & \mathbf{0} \\
-\mathbf{I}_{2} & \mathbf{I}_{2} & \mathbf{0} & \mathbf{0} & \mathbf{0} & \mathbf{0} & \mathbf{0} & \mathbf{0} \\
\mathbf{0} & -\mathbf{I}_{2} & \mathbf{I}_{2} & \mathbf{0} & \mathbf{0} & \mathbf{0} & \mathbf{0} & \mathbf{0} \\
\mathbf{0} & \mathbf{0} & -\mathbf{I}_{2} & \mathbf{I}_{2} & \mathbf{0} & \mathbf{0} & \mathbf{0} & \mathbf{0} \\
-\mathbf{I}_{2} & \mathbf{0} & \mathbf{0} & \mathbf{0} & 2 \mathbf{I}_{2} & \mathbf{0} & \mathbf{0} & -\mathbf{I}_{2} \\
\mathbf{0} & \mathbf{0} & \mathbf{0} & \mathbf{0} & -\mathbf{I}_{2} & \mathbf{I}_{2} & \mathbf{0} & \mathbf{0} \\
\mathbf{0} & \mathbf{0} & \mathbf{0} & \mathbf{0} & \mathbf{0} & -\mathbf{I}_{2} & \mathbf{I}_{2} & \mathbf{0} \\
\mathbf{0} & \mathbf{0} & \mathbf{0} & \mathbf{0} & \mathbf{0} & \mathbf{0} & -\mathbf{I}_{2} & \mathbf{I}_{2}
\end{array}\right]
$$

In other words, we transformed the $\mathbf{G}$ matrix to the conventional graph Laplacian matrix $\mathbf{L}$.

We recall the definition $[\mathbf{f}(\{\mathbf{x}\} ; \rho)]=\left[\mathbf{f}\left(\mathbf{x}_{1} ; \rho_{1}\right) ; \mathbf{f}\left(\mathbf{x}_{2} ; \rho_{2}\right) ; \cdots ;\right.$ $\left.\mathbf{f}\left(\mathbf{x}_{n} ; \rho_{n}\right)\right]$.

Since $\mathbf{T}[\mathbf{f}(\{\mathbf{x}\} ; \rho)]=\mathbf{T}\left[\mathbf{f}\left(\mathbf{T}^{-1}\{\mathbf{z}\} ; \rho\right)\right]$, we can find

$$
\begin{aligned}
\mathbf{T} & {[\mathbf{f}(\{\mathbf{x}\} ; \rho)]=\left[\frac{\rho_{1}}{\rho_{i}} \mathbf{R}\left(-\Delta_{1 j}\right) \mathbf{f}\left(\mathbf{x}_{i} ; \rho_{i}\right)\right] } \\
& =\left[\frac{\rho_{1}}{\rho_{i}} \mathbf{R}\left(-\Delta_{1 j}\right) \mathbf{f}\left(\frac{\rho_{i}}{\rho_{1}} \mathbf{R}\left(\Delta_{1 j}\right) \mathbf{z}_{i} ; \rho_{i}\right)\right]=\left[\mathbf{f}\left(\{\mathbf{z}\} ; \rho_{1} \mathbf{1}\right)\right] \\
& =\left[\mathbf{f}\left(\mathbf{z}_{1} ; \rho_{1}\right) ; \mathbf{f}\left(\mathbf{z}_{2} ; \rho_{1}\right) ; \cdots ; \mathbf{f}\left(\mathbf{z}_{n} ; \rho_{1}\right)\right]
\end{aligned}
$$

where we used $\mathbf{f}(\mathbf{R}(\Delta) \mathbf{x})=\mathbf{R}(\Delta) \mathbf{f}(\mathbf{x})$ and $\mathbf{f}(g \mathbf{x} ; \rho)=g \mathbf{f}(\mathbf{x} ; \rho / g)$ from Eqs. (2) and (3).

It is very important to notice that the radii of the final augmented Hopf oscillators in Eq. (16) are identical to $\rho_{1}$ such that $\rho=\rho_{1} \mathbf{1}$. Such a transformation to an identical oscillator of the same radius $\rho_{1}$ is indispensable for the following proof of synchronization. Equation (16) can be obtained only by exploiting the circular symmetry of the Hopf limit cycle, i.e., Eqs. (2) and (3).

By premultiplying Eq. (14) with $\mathbf{V}^{T}$ and substituting $\{\mathbf{z}\}=$ $\mathbf{V V}^{T}\{\mathbf{z}\}+\mathbf{1 1}^{T}\{\mathbf{z}\}$ into Eq. (16), we can obtain

$$
\mathbf{V}^{T}\{\dot{\mathbf{z}}\}=\mathbf{V}^{T}\left[\mathbf{f}\left(\mathbf{V} \mathbf{V}^{T}\{\mathbf{z}\}+\mathbf{1 1}^{T} / n\{\mathbf{z}\} ; \rho_{1} \mathbf{1}\right)\right]-k(t) \mathbf{V}^{T} \mathbf{L} \mathbf{V} \mathbf{V}^{T}\{\mathbf{z}\}
$$

where we used $\mathbf{L} \mathbf{1 1}{ }^{T}=0$.

We can construct the following virtual dynamics of $\mathbf{y}$ from the preceding equation

$$
\dot{\mathbf{y}}=\mathbf{V}^{T}\left[\mathbf{f}\left(\mathbf{V y}+\mathbf{1 1}^{T} / n\{\mathbf{z}\} ; \rho_{1} \mathbf{1}\right)\right]-k(t) \mathbf{V}^{T} \mathbf{L} \mathbf{V y}
$$

which has $\mathbf{y}=\mathbf{V}^{T}\{\mathbf{z}\}$ as one particular solution.

The other particular solution of Eq. (18) is $\mathbf{y}=\mathbf{0}$, since $\mathbf{V}^{T}\left[\mathbf{f}\left(\mathbf{1 1}^{T} / n\{\mathbf{z}\} ; \rho_{1} \mathbf{1}\right)\right]=\mathbf{0}$. If the virtual system $\mathbf{y}$ in Eq. (18) is contracting, then the two particular solutions tend to each other exponentially from any initial condition, thereby completing the proof.

The virtual system Eq. (18) is contracting if $\mathbf{V}^{T}[\mathbf{f}] \mathbf{V}-$ $k \mathbf{V}^{T}\left(\mathbf{L}+\mathbf{L}^{T}\right) \mathbf{V} / 2<0$ due to Theorem 1. This condition is equivalent to $k \lambda_{\min }\left(\mathbf{V}^{T}\left(\mathbf{L}+\mathbf{L}^{T}\right) \mathbf{V} / 2\right)>\lambda$, since the maximum eigenvalue $\lambda_{\max }\left(\mathbf{V}^{T}[\mathbf{f}] \mathbf{V}\right) \leq \lambda$. For the example network in Fig. 3a, this condition corresponds to $k>\lambda / 0.198$. This also holds for a time-varying $k(t)$, if the condition holds uniformly for all time.

The same proof works for an arbitrary CPG network on a balanced graph that has $\mathbf{V}^{T}\left(\mathbf{L}+\mathbf{L}^{T}\right) \mathbf{V} / 2>0$. For undirected graphs (all the connections are bidirectional), $\mathbf{L}$ automatically becomes a balanced symmetric matrix.

In conclusion, Theorem 2 can be used to find the proper coupling strength $k$ to exponentially and globally stabilize the coupled Hopf oscillators given in Eq. (4).

\section{Fast Inhibition of Oscillation by Hopf Bifurcation}

As stated earlier, we can rapidly inhibit the oscillatory motion of the coupled Hopf oscillators in Eq. (4) by exploiting the bifurcation property of the Hopf oscillator model. In other words, changing the $\sigma=1$ in Eq. (1) to $\sigma=-1$ would rapidly convert the limit-cycle dynamics to exponentially stable dynamics converging to the origin such that $u \rightarrow a$ and $v \rightarrow 0$. This single bifurcation parameter $\sigma$ can be used to switch the flapping-flight mode to the gliding mode or soaring mode without dramatically changing the CPG oscillator network. Simulation results that alternate between two different flight modes are presented in Sec. IV.

Theorem 3: For any positive gain $\bar{k}>0$, any initial condition $\{\mathbf{x}\}$ of the coupled Hopf model with $\sigma=-1$ given in Eq. (4) converges to the origin $(\{\mathbf{x}\} \rightarrow \mathbf{0})$ such that $u_{i} \rightarrow a_{i}$ and $\overline{v_{i}} \rightarrow 0$ for all $i=1, \ldots, n$. The oscillation frequency $\omega$ need not change to zero.

Proof 3: It is straightforward to show that $\sigma=-1$ will cause the uncoupled Hopf oscillator in Eq. (1) to have exponentially stable dynamics for any $(u, v)$ except the shifted origin $(a, 0)$, since the symmetric part of the Jacobian $\mathbf{F}$ in Eq. (8) is now strictly negative definite, regardless of any $\omega$. Thus, any positive $k$ will lead to exponentially synchronizing dynamics that tend exponentially to the shifted origin $\left(a_{i}, 0\right)$, and this can be shown, as was shown for the proof of Theorem 2 .

We can also turn the limit-cycle dynamics to the dynamics with a stable equilibrium by changing the coupling gains, described as fast inhibition in [6]. However, the method using bifurcation is superior in the sense that we can keep the original coupling gains and Laplacian matrices for alternating flight modes. It should be noted that changing $\omega$ to zero would also result in no reciprocal flapping motion; however, the converged steady-state value depends on the initial conditions, whereas $\sigma=-1$ would always make the system converge to the desired value $\left(a_{i}, 0\right)$ for each joint $i$.

\section{E. Perspectives on Sensory Feedback Connection}

The property of robustness, inherent in the CPG-based control, is particularly emphasized by the literature (see [68]). Stable locomotion can be achieved using the interaction between the CPG model, the physical model of the body, and the environment [69]. Most models [45,54,58] use an open-loop approach without sensor feedback, and others $[64,70]$ incorporate sensor feedback to modulate the reference oscillator patterns. One drawback is that such open-loop approaches do not ensure the synchronization of the physical states in the presence of external disturbances. In other words, the mutual entrainment $[52,69,71]$ between the CPG and the mechanical body is not guaranteed. Recently, two new closed-loop CPG control strategies that reinforce emerging rhythmic patterns of actual physical joints such as foil-fin actuators have been proposed $[50,51]$. Such a reflex-based closed-loop CPG method has a potential for discovering practical ways of flapping-wing coordination in the presence of external disturbances. For example, we can modify Eq. (4) to incorporate actual states of actuators:

$$
\dot{\mathbf{x}}_{i}=\mathbf{f}\left(\mathbf{x}_{i} ; \rho_{i}\right)-k(t) \sum_{j \in \mathcal{N}_{i}}^{m_{i}}\left(\mathbf{p}_{i}-\frac{r_{i}}{r_{j}} \mathbf{R}\left(\Delta_{i j}\right) \mathbf{p}_{j}\right)
$$

where $\mathbf{p}_{i}$ and $\mathbf{p}_{j}$ are the actual actuator joint angles measured directly from sensors, and $r_{i} / r_{j}$ denotes the new amplitude scaling for actuators. Then, as with Theorem 2, we can prove the stability of Eq. (19) by assuming that the actuator states can be represented by $\mathbf{p}_{i}=\bar{p}_{i}(t) \mathbf{R}\left(\Delta_{i, \text { lag }}\right) \mathbf{x}_{i}$, where $\Delta_{i, \text { lag }}$ indicates a time delay between $\mathbf{p}_{i}$ and $\mathbf{x}_{i}$. Readers are referred to [50,51] for details.

In Sec. III, we present the wing kinematic model and the dynamic model of flapping-flight dynamics that can be driven by the CPG network.

\section{Wing Kinematics, Aerodynamic Forces, and Vehicle Dynamics}

We derive a three-dimensional model of the wing kinematics in this section. The wing kinematic model supports both flexible and 


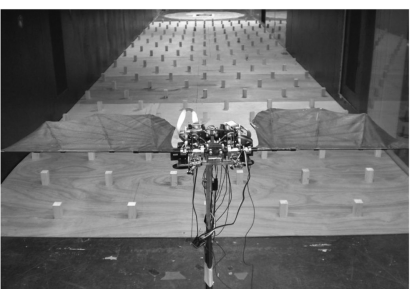

a)

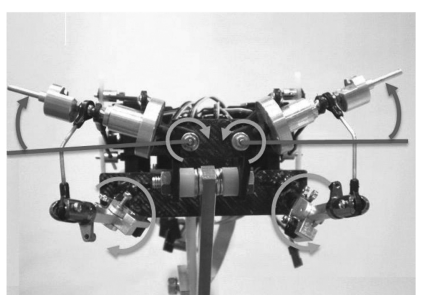

b)

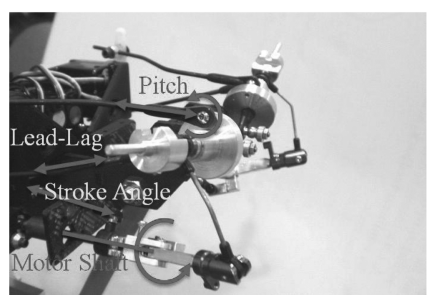

c)

Fig. 4 An 8-DOF robotic bat (with 10 control variables) MAV [25].

rigid wings. We also present the 6-DOF dynamic equations of motion of flapping flight that can be used to validate the coupled wing control driven by CPG. We also show that the effective angle of attack of each blade element can be effectively controlled by the synchronized pitching $\theta_{w}$ control as well as the wing-joint angles such as $\phi_{w}$ and $\psi_{w}$.

We present a realistic modeling that encompasses a tilted stroke angle, the lead-lag motion, and the relative body velocity, in addition to the stroke and pitch angles. In deriving these equations, the actual control degrees of freedom of the robotic bat MAV testbed shown in Fig. 4 are considered. Its half-wingspan is $32 \mathrm{~cm}$. The 8-DOF robotic bat has 10 independent control variables, including separate flapping frequency and amplitude control for each wing as well as pitch and lead-lag angle servos (see [25] for details).

Figure 5a shows a side view of the flapping-flying MAV with the body frame $\mathbf{x}_{b}=\left(x_{b}, y_{b}, z_{b}\right)^{T}$ and the stroke-plane frame $\mathbf{x}_{s}=$

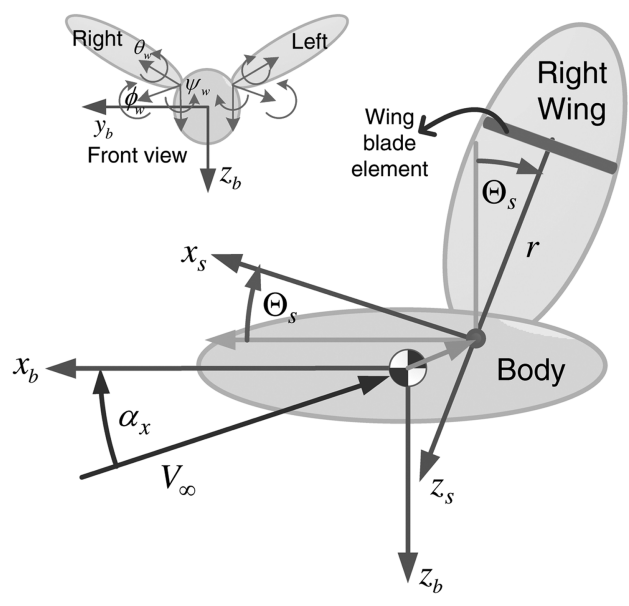

a) Transformation from the vehicle body frame to the stroke plane frame of the right wing

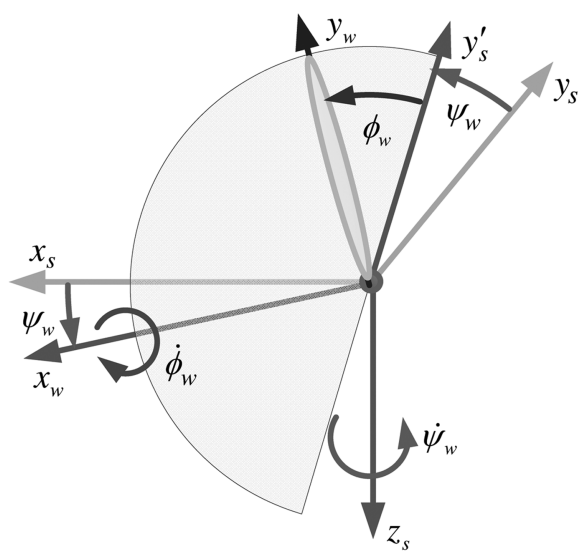

b) Transformation from the stroke plane frame to the wing frame

Fig. 5 Schematic of the 3-D wing motions. $\left(x_{s}, y_{s}, z_{s}\right)^{T}$ of the right wing. As with the robotic bat in Fig. 4 , we assume that each wing has flapping $\phi_{w}$, pitch $\theta_{w}$, and lead-lag $\psi_{w}$ control. We develop only the equations of the right wing, since similar expressions for the left wing can straightforwardly follow. The center of the stroke-plane frame is located at $\left(d_{x}, d_{y}, d_{z}\right)$, and it is tilted by the inclination angle $\Theta_{s}(t)$, which can be a function of time and the forward velocity. Without the lead-lag motion, the axes $y_{s}$ and $z_{s}$ define the stroke plane. Hence, the transformation between these coordinate axes can be given by

$$
\mathbf{x}_{b}=\mathbf{T}_{b s}\left(\Theta_{s}\right) \mathbf{x}_{s}+\left(d_{x}, d_{y}, d_{z}\right)^{T}
$$

where

$$
\mathbf{T}_{b s}\left(\Theta_{s}\right)=\left[\begin{array}{ccc}
\cos \Theta_{s} & 0 & \sin \Theta_{s} \\
0 & 1 & 0 \\
-\sin \Theta_{s} & 0 & \cos \Theta_{s}
\end{array}\right]
$$

In this paper, $\mathbf{T}_{b s}$ denotes the transformation from $\mathbf{x}_{s}$ to $\mathbf{x}_{b}$, whereas $\mathbf{T}_{s b}=\mathbf{T}_{b s}^{T}$ would correspond to the transformation from $\mathbf{x}_{b}$ to $\mathbf{x}_{s}$.

For a hovering insect, the stroke plane is almost horizontal (i.e., $\Theta_{s}=90 \mathrm{deg}$ in our coordinate definition in Fig. 5a), resulting in forward and backward reciprocating motions. This is the assumption used for some prior work [7,20,23,24]. In contrast, the stroke angle of birds and bats varies as a function of flight speed; at a low speed, the angle is almost horizontal $\left(\Theta_{s}=90 \mathrm{deg}\right)$ and it approaches $\Theta_{s}=$ 0 deg as the flight speed increases.

If there is no lead-lag motion, the additional transformation for a wing stroke angle $\phi_{w}$ would complete all the required transformation between the body frame and the wing frame. However, a nonzero lead-lag angle further complicates the wing kinematics. Choosing the rotational axes for flapping, lead lag, and pitch depends on the actual hardware setup and actuators, and our choice is influenced by the robotic bat MAV shown in Fig. 4 (see [25]). In contrast with Azuma's derivation [3], in which the stroke angle $\Theta_{s}(t)$ is dependent on the $\phi_{w}(t)$ and the lead-lag angle $\psi_{w}(t)$, our $\Theta_{s}(t)$ is an independent control variable. Our decision is based on the observation that $\Theta_{s}(t)$ can be an important control variable for efficient engineered flapping flight. Further, this kind of actuator mechanism is easier to implement and control. As shown in Fig. $\underline{5 b}$, the lead-lag angle is defined by the rotation about the $z_{s}$ axis: the $z$ axis in the stroke-plane frame. In contrast with the fixed angle rotation in [3], we then rotate about the new $x$ axis to obtain the wing frame $\mathbf{x}_{w}$. For both wings, the positive direction of $\psi_{w}$ is the forward direction, and the positive stroke angle $\phi_{w}$ indicates an upstroke motion. This sign convention does not agree with the original positive direction of rotation for the right wing, so extra care should be taken to determine the correct angular transformation matrices.

For the right wing, the transformation between the stroke-plane frame $\mathbf{x}_{s}$ and the wing frame $\mathbf{x}_{w}$ can be written as

$$
\begin{aligned}
\mathbf{x}_{s} & =\mathbf{T}_{s w}\left(\phi_{w}, \psi_{w}\right) \mathbf{x}_{w} \\
& =\left[\begin{array}{ccc}
\cos \psi_{w} & \sin \psi_{w} & 0 \\
-\sin \psi_{w} & \cos \psi_{w} & 0 \\
0 & 0 & 1
\end{array}\right]\left[\begin{array}{ccc}
1 & 0 & 0 \\
0 & \cos \phi_{w} & \sin \phi_{w} \\
0 & -\sin \phi_{w} & \cos \phi_{w}
\end{array}\right] \mathbf{x}_{w}
\end{aligned}
$$

To compute the local lift and drag of a blade element with width $\mathrm{d} r$ and wingspan coordinate $r \in[0, R]$, we need to transform the 
velocities in body coordinates to the incident velocities in the rotated wing frame. For example, consider the vehicle body speed $V_{b}$ with the body angle of attack $\alpha_{x}$ and the side-slip angle $\alpha_{y}$. Note that $\alpha_{y}$ is commonly denoted by $\beta$ in the aerospace community, but in this paper, $\beta$ denotes the direction of the relative wind of a blade element. Then the freestream velocity in the body frame can be written as

$$
\mathbf{V}_{b}=\left(V_{b} \cos \alpha_{y} \cos \alpha_{x}, V_{b} \sin \alpha_{y}, V_{b} \cos \alpha_{y} \sin \alpha_{x}\right)^{T}+\mathbf{v}_{i}+\mathbf{v}_{E}
$$

where $\mathbf{v}_{i}$ and $\mathbf{v}_{E}$ denote the induced velocity vector and the wind velocity vector, respectively. In other words, in the absence of $\mathbf{v}_{i}$ and $\mathbf{v}_{E}$, the vector $\mathbf{V}_{b}$ equals the velocity of the vehicle in the body frame. Let us assume that $\alpha_{x}$ and $\alpha_{y}$ include the effects of the induced velocity and $\mathbf{v}_{E}$ is small.

Then the free-velocity vector $\mathbf{V}_{b}$ in the body frame can be transformed to the wing frame. In addition, we can also compute the additional velocity on the wing frame induced from the body angular rate $\boldsymbol{\Omega}_{b}=(p, q, r)^{T}$ and the offset distance $\mathbf{d}=\left(d_{x}, d_{y}, d_{z}\right)^{T}$ of the stroke-plane frame (see Fig. 5 a). By adding these two terms, we can obtain

$$
\mathbf{V}_{b}^{w}=\mathbf{T}_{w s}\left(\phi_{w}, \psi_{w}\right) \mathbf{T}_{s b}\left(\Theta_{s}\right)\left(\mathbf{V}_{b}+\boldsymbol{\Omega}_{b} \times \mathbf{d}\right)
$$

To compute the rotational velocity on the wing frame produced by the flapping $\phi_{w}$ and lead-lag $\psi_{w}$ motions, as well as a relatively slower stroke-angle change $\Theta_{s}(t)$, it is more convenient to construct the angular rate vector in the stroke-plane frame as follows:

$$
\boldsymbol{\Omega}_{\mathrm{tot}}=\mathbf{T}_{s b}\left(\Theta_{s}\right) \boldsymbol{\Omega}_{b}+\left(\begin{array}{c}
-\cos \psi_{w} \dot{\phi}_{w} \\
\sin \psi_{w} \dot{\phi}_{w}+\dot{\Theta}_{s} \\
-\dot{\psi}_{w}
\end{array}\right)
$$

Then we can compute the induced rotational velocity from the wing motions of the blade element $\mathrm{d} r$ :

$$
\mathbf{V}_{\mathrm{rot}}^{w}=\left(\mathbf{T}_{w s}\left(\phi_{w}, \psi_{w}\right) \boldsymbol{\Omega}_{\mathrm{tot}}\right) \times\left(\left(\begin{array}{c}
0 \\
r \\
0
\end{array}\right)+\left(\begin{array}{c}
x_{w}(r) \\
y_{w}(r) \\
z_{w}(r)
\end{array}\right)\right)+\left(\begin{array}{c}
\dot{x}_{w}(r) \\
\dot{y}_{w}(r) \\
\dot{z}_{w}(r)
\end{array}\right)
$$

where $x_{w}(r), y_{w}(r)$, and $z_{w}(r)$ are the deformations of the blade element due to aeroelastic deformation or active cambering control that can be found in bat flight. Hence, the derivations in this section can be used for flexible-wing models, although the $C_{L}(\alpha)$ and $C_{D}(\alpha)$ functions should be corrected for such cambered wing shapes.

By adding $\mathbf{V}_{b}^{w}$ in Eq. (23) and $\mathbf{V}_{\text {rot }}^{w}$ in Eq. (25), we can obtain the total velocity of the wind at the blade element, located at $r$ on the wingspan axis:

$$
\begin{aligned}
& \mathbf{V}_{w}=\left(\begin{array}{c}
V_{w x} \\
V_{w y} \\
V_{w z}
\end{array}\right)=\mathbf{T}_{w s}\left(\phi_{w}, \psi_{w}\right) \mathbf{T}_{s b}\left(\Theta_{s}\right)\left(\mathbf{V}_{b}+\boldsymbol{\Omega}_{b} \times \mathbf{d}\right) \\
& +\left(\mathbf{T}_{w s}\left(\phi_{w}, \psi_{w}\right) \boldsymbol{\Omega}_{\mathrm{tot}}\right) \times\left(\left(\begin{array}{c}
0 \\
r \\
0
\end{array}\right)+\left(\begin{array}{c}
x_{w}(r) \\
y_{w}(r) \\
z_{w}(r)
\end{array}\right)\right)+\left(\begin{array}{c}
\dot{x}_{w}(r) \\
\dot{y}_{w}(r) \\
\dot{z}_{w}(r)
\end{array}\right)
\end{aligned}
$$

A similar expression can be obtained for the left wing. The preceding derivations can also be straightforwardly extended to account for the second joint (elbow) if each wing has one.

We can now obtain the local effective angle of attack $\alpha_{w}$ of the blade element to determine aerodynamic forces and torque. Let us assume that the deformation of a rigid wing is negligible and there is no active cambering control. The contribution from the body angular rate $\boldsymbol{\Omega}_{b}$ is also small. Equation (26) then reduces to

$$
\begin{aligned}
& \left(\begin{array}{c}
V_{w x} \\
V_{w y} \\
V_{w z}
\end{array}\right)=\mathbf{T}_{w s}\left(\phi_{w}, \psi_{w}\right) \mathbf{T}_{s b}\left(\Theta_{s}\right) \mathbf{V}_{b} \\
& +\left(\mathbf{T}_{w s}\left(\phi_{w}, \psi_{w}\right)\left(\begin{array}{c}
-\cos \psi_{w} \dot{\phi}_{w} \\
\sin \psi_{w} \dot{\phi}_{w}+\dot{\Theta}_{s} \\
-\dot{\psi}_{w}
\end{array}\right)\right) \times\left(\begin{array}{l}
0 \\
r \\
0
\end{array}\right)
\end{aligned}
$$

We can now obtain the local incident angle $\beta_{w}$ (measured clockwise), the angle of attack $\alpha_{w}$, and the speed of the wind $V_{r}$ on the blade element on the right wing as follows:

$$
\begin{gathered}
\beta_{w}(r, t)=\tan ^{-1} \frac{-V_{w z}}{V_{w x}}, \quad \alpha_{w}(r, t)=\theta_{w}(t)-\beta_{w}(r, t) \\
V_{r}^{2}(r, t)=\sqrt{V_{w x}^{2}+V_{w z}^{2}}
\end{gathered}
$$

where we neglected the flow along the wingspan $V_{w y}$, and the wing rotation $\theta_{w}(t)$ controller can be properly designed to yield a positive angle of attack for both upstroke and downstroke motions. If we consider an aeroelastic rotation of the wing, $\theta_{w}(t)$ can capture both the active and passive rotation of each blade element.

If the MAV were flying with a zero flight-path angle and $\Theta_{s}=\psi_{w}=0$, we could obtain

$$
\beta_{w}(r, t)=\tan ^{-1} \frac{r \dot{\phi}_{w}}{V_{b}}=\tan ^{-1} \frac{2 r k_{r}}{c}, \quad k_{r}=\frac{\dot{\phi}_{w} c}{2 V_{b}}
$$

where the reduced frequency $k_{r}$ compares the velocity by the wing flapping motion with the forward speed, thereby indicating the degree of unsteady aerodynamics (if $k_{r} \gg 1$, unsteady effects dominate $[2,19])$.

We can see that the sign of $\beta_{w}$ is consistent with the positive direction of the flapping (stroke) angle $\phi_{w}$, since the downstroke $\dot{\phi}_{w}<0$ leads to the negative flow angle $\beta_{w}<0 ; \beta_{w}$ also becomes a function of $\dot{\phi}_{w}$, indicating why the pitch angle $\theta_{w}$ should have a $90 \mathrm{deg}$ phase difference with $\phi_{w}\left(\Delta_{21}=90 \mathrm{deg}\right)$ to maximize the local angle of attack. Figure 6 shows a result of the wind-tunnel test of the robotic bat shown in Fig. 4. The robotic bat is mounted horizontally in a wind tunnel [25]. In Fig. 6, the pitch motion $\theta_{w}$ was activated in $30 \mathrm{~s}$ with about $\Delta_{21}=90 \mathrm{deg}$. This increased the lift $F_{z}$ by more than a factor of 2 , whereas the wind speed and the CPG frequency $\omega$ were held constant. This result shows that the synchronized pitch control is indispensable. We can find the most efficient phase difference $\left(\Delta_{21}\right)$, which can be compared with Dickinson et al.'s [20] experimentation with a robot fly (advanced rotation with $\Delta_{21}=90+\delta \mathrm{deg}$ ).

Once we obtain the local effective angle of attack $\alpha_{w}$, we can proceed to obtain the aerodynamic forces of the blade element by evaluating the lift and drag coefficients $C_{L}\left(\alpha_{w}\right)$ and $C_{D}\left(\alpha_{w}\right)$. Flapping flight, typically within a low-Reynolds-number regime $\left(\operatorname{Re}<10^{5}\right)$, is governed by unsteady aerodynamics characterized by large-scale vortex structures. It is understood that the main liftenhancement mechanism of flapping flight is governed by 1) the leading-edge vortex that leads to delayed stall at a very high angle of attack, 2) the rotational circulation lift, and 3) wake capture that

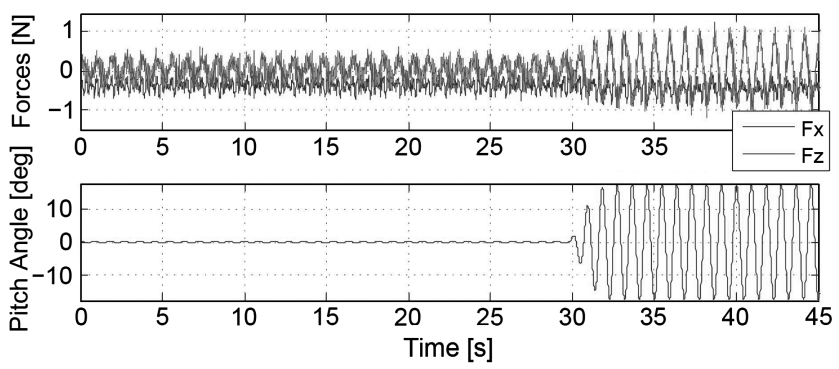

Fig. 6 Impact of the synchronized pitch $\theta_{w}$ oscillation. 
generate aerodynamic forces during flapping-angle reversals [20]. In particular, Dickinson et al.'s series of papers [7,20], by crossvalidating the numerical computation and experimentation using the Robofly, shows that a quasi-steady aerodynamic model predicts the aerodynamic coefficients reasonably well. Although computational fluid dynamics is much too computationally burdensome to justify its use in control design, this quasi-steady approximation method can be verified and improved by the experimental setup, such as the robotic bat described in [25].

The seminal paper by Dickinson et al. [20] used a hovering pair of wings without a forward speed as follows:

$$
\begin{aligned}
& C_{L}\left(\alpha_{w}\right)=0.225+1.58 \sin \left(2.13 \alpha_{w}-7.2 \mathrm{deg}\right) \\
& C_{D}\left(\alpha_{w}\right)=1.92-1.55 \cos \left(2.04 \alpha_{w}-9.82 \mathrm{deg}\right)
\end{aligned}
$$

It should be noted that Dickinson et al.'s [ㄱ,20] robot-fly setup used a horizontal stroke plane, as typically seen in insect flight, whereas we assume a $90 \mathrm{deg}$ stroke-plane angle. The angle $\alpha_{w}$ for a general flapping wing is time-varying, as described in this section. A recent paper [7] considers a nonzero forward speed, and the coefficients of Eq. (30) can be modified to become functions of the reduced frequency $k_{r}$.

From the quasi-steady approximation, we can compute the lift and drag forces acting on the blade element with width $\mathrm{d} r$ as follows:

$$
\begin{aligned}
\mathrm{d} L & =\frac{1}{2} \rho C_{L}\left(\alpha_{w}(r, t)\right) c(r) V_{r}^{2}(r, t) \mathrm{d} r \\
\mathrm{~d} D & =\frac{1}{2} \rho C_{D}\left(\alpha_{w}(r, t)\right) c(r) V_{r}^{2}(r, t) \mathrm{d} r
\end{aligned}
$$

In addition, Ellington [8-13] derived the wing circulation $\Gamma_{r}=$ $\pi \dot{\alpha} c^{2}\left(3 / 4-\hat{x}_{0}\right)$ based on the Kutta-Joukowski condition. This quasi-steady approximation for the rotational lift can be written as

$$
\mathrm{d} L_{\mathrm{rot}}=\frac{1}{2} \rho\left(2 \pi\left(\frac{3}{4}-\hat{x}_{0}\right)\right) c^{2}(r) V_{r}(r, t) \dot{\alpha}_{w} \mathrm{~d} r
$$

where $\hat{x}_{0}$ is the location of the pitch axis along the mean chord length, and $\dot{\alpha}_{w}$ can be computed from Eq. (28) and often approximated reasonably well by the angular rate of the wing pitch motion $\dot{\theta}_{w}$.

The total $x$ and $z$ directional forces of a single wing (either right or left) in the body frame are obtained as

$$
\begin{gathered}
F_{w z}=\int_{r=0}^{R} \mathrm{~d} D \sin \beta_{w}-\left(\mathrm{d} L+\mathrm{d} L_{\mathrm{rot}}\right) \cos \beta_{w} \\
F_{w x}=\int_{r=0}^{R}-\left(\mathrm{d} L+\mathrm{d} L_{\mathrm{rot}}\right) \sin \beta_{w}-\mathrm{d} D \cos \beta_{w}
\end{gathered}
$$

where the positive direction of $z_{b}$ is downward, as shown in Fig. 5a.

The $F_{w x}$ and $F_{w z}$ forces on the wing frame given in Eq. (33) can be transformed into the forces in the vehicle body frame:

$$
\mathbf{F}_{\text {right }}=\left(\begin{array}{c}
F_{x} \\
F_{y} \\
F_{z}
\end{array}\right)_{\text {right }}=\mathbf{T}_{b s}\left(\Theta_{s}\right) \mathbf{T}_{s w}\left(\phi_{w}, \psi_{w}\right)\left(\begin{array}{c}
F_{w x} \\
0 \\
F_{w z}
\end{array}\right)_{\text {right }}
$$

where we added the subscript right to indicate the right wing. A similar expression can be obtained for the left wing $\left(\mathbf{F}_{\text {left }}\right)$. Each wing has different wing angular parameters such as $\phi_{w}, \psi_{w}$, and $\theta_{w}$, although the stroke-plane angle $\Theta_{s}$ is the same for both wings.

To compute the rotational moments generated by the aerodynamic forces, we first calculate the position of the wing blade element with respect to the body frame:

$$
\mathbf{p}(r)=\mathbf{T}_{b s}\left(\Theta_{s}\right) \mathbf{T}_{s w}\left(\phi_{w}, \psi_{w}\right)\left(\begin{array}{c}
0 \\
r \\
0
\end{array}\right)+\left(\begin{array}{c}
d_{x} \\
d_{y} \\
d_{z}
\end{array}\right)
$$

Then we can compute the aerodynamic moments with respect to the c.g.:

$$
\begin{aligned}
& \left(\begin{array}{l}
\mathrm{d} M_{x} \\
\mathrm{~d} M_{y} \\
\mathrm{~d} M_{z}
\end{array}\right)=\mathbf{p}(r) \times\left(\mathbf{T}_{b s}\left(\Theta_{s}\right) \mathbf{T}_{s w}\left(\phi_{w}, \psi_{w}\right)\right. \\
& \left.\quad \times\left(\begin{array}{c}
-\left(\mathrm{d} L+\mathrm{d} L_{\text {rot }}\right) \sin \beta_{w}-\mathrm{d} D \cos \beta_{w} \\
0 \\
\mathrm{~d} D \sin \beta_{w}-\left(\mathrm{d} L+\mathrm{d} L_{\text {rot }}\right) \cos \beta_{w}
\end{array}\right)\right)+\left(\begin{array}{c}
\mathrm{d} M_{x 0} \\
\mathrm{~d} M_{y 0} \\
\mathrm{~d} M_{z 0}
\end{array}\right)
\end{aligned}
$$

$$
\begin{aligned}
& \left(\begin{array}{c}
\mathrm{d} M_{x 0} \\
\mathrm{~d} M_{y 0} \\
\mathrm{~d} M_{z 0}
\end{array}\right)=\mathbf{T}_{b s}\left(\Theta_{s}\right) \mathbf{T}_{s w}\left(\phi_{w}, \psi_{w}\right) \mathbf{T}_{\theta_{w}}\left(\theta_{w}\right) \frac{1}{2} \rho V_{r}^{2} c(r) \mathrm{d} r \\
& \quad \times\left(\begin{array}{c}
r C_{l 0} \\
r(r)\left(C_{m 0}+C_{m \alpha, w} \alpha_{w}\right) \\
r C_{n 0}
\end{array}\right) \\
& M_{x}=\int_{r=0}^{R} \mathrm{~d} M_{x}, \quad M_{y}=\int_{r=0}^{R} \mathrm{~d} M_{y}, \quad M_{z}=\int_{r=0}^{R} \mathrm{~d} M_{z}
\end{aligned}
$$

where $\mathrm{d} M_{x 0}, \mathrm{~d} M_{x 0}$, and $\mathrm{d} M_{x 0}$ denote the constant aerodynamic moments that include the moment at the mean aerodynamic center, computed by the moment coefficients $C_{l 0}, C_{m 0}, C_{m \alpha, w}$, and $C_{n 0}$. The transformation matrix $\mathbf{T}_{\theta_{w}}\left(\theta_{w}\right)$ rotates the wing frame about the $y_{w}$ axis by the wing pitch rotation angle $\theta_{w}$.

By combining all the forces and moments from the right wing and the left wing, we can derive 6-DOF equations of motion for the flapping-flying MAV in the body frame, for which the orientation with respect to the inertial frame is described by the Euler angles. We assume the mass and the moment of inertia of the wing compared with the body weight are negligible so that the c.g. remains fixed. The translational motion of the c.g. of the vehicle driven by the aerodynamic force terms in Eq. (34) can be expressed as

$$
m \dot{\mathbf{V}}_{b}+m \boldsymbol{\Omega}_{b} \times \mathbf{V}_{b}=\mathbf{T}_{b e}\left(\phi_{b}, \theta_{b}, \psi_{b}\right) \mathbf{F}_{g}+\mathbf{F}_{\text {right }}+\mathbf{F}_{\text {left }}+\mathbf{A}
$$

Each wing has different wing angular parameters such as $\phi_{w}, \psi_{w}$, and $\theta_{w}$, although the stroke-plane angle $\Theta_{s}$ is the same for each wing.

The equations of rotational motion are driven by the aerodynamic moments $\mathbf{M}_{\text {right }}$ and $\mathbf{M}_{\text {left }}$ of each wing from Eq. (36):

$$
\mathbf{I}_{b} \boldsymbol{\Omega}_{b}+\boldsymbol{\Omega}_{b} \times\left(\mathbf{I}_{b} \boldsymbol{\Omega}_{b}\right)=\mathbf{M}_{\text {right }}+\mathbf{M}_{\mathrm{left}}+\mathbf{B}
$$

The relationship between the body angular rate $\boldsymbol{\Omega}_{b}=(p, q, r)^{T}$ and the Euler angle vector $\mathbf{q}_{b}=\left(\phi_{b}, \theta_{b}, \psi_{b}\right)^{T}$ can be determined by [25]

$$
\dot{\mathbf{q}}_{b}=\mathbf{Z}\left(\mathbf{q}_{b}\right) \boldsymbol{\Omega}_{b}
$$

where any other orientation representations such as quaternions can be used in lieu of the Euler angles in the preceding equations. Any disturbance force and torque can also be added to the equations.

\section{CPG-Based Flapping Flight Control and Simulation Results}

The aim of this section is to show that CPG-based flight control can stabilize and control flapping-flight dynamics given in Sec. III by commanding a reduced set of CPG parameters that generate the phase-synchronized or symmetry-breaking oscillatory motions of two main wings. In particular, we show that the dynamics can be controlled without a tail or aerodynamic control surfaces such as ailerons, elevators, rudders, and directional control of tail wings. Another important contribution is to demonstrate stable transitions between gliding and flapping-flight modes by using the Hopf bifurcation. 


\section{A. CPG-Based Flapping Flight Control}

The example presented in this paper has three different flight modes: gliding, flapping, and turning. We show that only three control laws for $\omega(t)$, the symmetric phase difference $\left(\Delta_{32}=\Delta_{76}\right)$ between $\theta_{w}$ and $\psi_{w}$, and symmetric breaking of the lead-lag maximum amplitudes $\left(\rho_{3} \neq \rho_{7}\right)$ can control both longitudinal and lateral dynamics of tailless flapping flight with six independent wingjoint angles.

\section{Flapping Flight Control by Flapping Frequency}

We propose a novel control law unique to our CPG setup that reduces control dimensionality to only three parameters. The first parameter is the oscillation frequency $\omega(t)$ of the coupled Hopf oscillators in Eq. (4). The flapping frequency $\omega(t)$ correlates with increased lift and thrust. Those in turn correlate with the velocity of the body. For example, we can consider the following control law,

$$
\omega(t)=\int_{0}^{t} \dot{\omega} \mathrm{d} t=K_{\omega} \int_{0}^{t}\left(V_{x, \text { desired }}-V_{x, \text { actual }}\right) \mathrm{d} t
$$

and use the following corollary.

Corollary 1: From the dynamic equation of the Hopf oscillator in Eqs. (1) and (4), any time-varying $\omega(t)$ does not affect the synchronization stability proof for Theorem 2 .

Proof 4: The symmetric part of $\mathbf{f}$ cancels the $\omega(t)$ term and $\omega(t)$ does not change the maximum eigenvalue of $\mathbf{V}^{T}[\mathbf{f}] \mathbf{V}$. The rest of the proof follows Theorem 2 .

\section{Flapping Flight Control by Phase Differences and Symmetry Breaking}

The phase differences $\Delta_{i j}$ ) of the coupled Hopf oscillators in Eq. (4) can be effective control mechanisms for flapping-flight dynamics, and this controllability is another reason that justifies our coupled nonlinear oscillator framework rather than individual control of each angle. However, our synchronization stability proof in Theorem 2 assumes constant or relatively slowly varying phase differences. Our previous work [25] showed that the synchronization error terms from time-varying $\overline{\Delta_{i j}}(t)$ are bounded and can be made arbitrarily small with a sufficiently large coupling gain $k$ due to robust contraction analysis [67]. In the present paper, we show that the synchronization errors due to time-varying $\Delta_{i j}(t)$ and $\rho_{i}(t)$ can tend exponentially to zero, thereby further strengthening our claim with the coupled Hopf oscillators.

Corollary 2: Let us assume that the synchronization condition of $k$ in Theorem 2 holds. For time-varying phase differences $\Delta_{i j}(t)$ or time-varying radii $\rho_{i}(t)$ and $\rho_{j}(t)$, the synchronization errors of the rotated Hopf states $\{\mathbf{z}\}$ globally and exponentially converge to zero, i.e., $\mathbf{V}^{T}\{\mathbf{z}\} \rightarrow \mathbf{0}$, if we add the additional time-varying term $-\mathbf{T}^{-1} \mathbf{T}\{\mathbf{x}\}$ to the coupled Hopf oscillator dynamics in Eq. (6) such that

$$
\{\dot{\mathbf{x}}\}=[\mathbf{f}(\{\mathbf{x}\} ; \rho)]-k(t) \mathbf{G}\{\mathbf{x}\}-\mathbf{T}^{-1} \dot{\mathbf{T}}\{\mathbf{x}\}
$$

where the block-diagonal transformation matrix $\mathbf{T}\left(\Delta_{i j}, \rho_{i}\right)$ is defined such that $\{\mathbf{z}\}=\mathbf{T}\{\mathbf{x}\}$ as in Eq. (11), and $\dot{\mathbf{T}}=(\mathrm{d} / \mathrm{d} t) \mathbf{T}\left(\Delta_{i j}, \rho_{i}\right)$ is a function of $\dot{\Delta}_{i j}$ and $(\mathrm{d} / \mathrm{d} t)\left(\rho_{i} / \rho_{j}\right)$ for each time-varying $\Delta_{i j}, \rho_{i}$, and $\rho_{j}$.

As shall be seen later, we do not have to directly compute the time derivatives of $\Delta_{i j}(t)$ and $\rho_{i}(t) / \rho_{j}(t)$ if we select the control laws carefully.

Proof 5: Since $\mathbf{T}\{\mathbf{x}\}=\{\mathbf{z}\}$, we can verify $\{\dot{\mathbf{z}}\}=\dot{\mathbf{T}}\{\mathbf{x}\}+\mathbf{T}\{\dot{\mathbf{x}}\}$. Hence, premultiplying Eq. (43) by $\mathbf{T}$ results in the same dynamics of $\{\mathbf{z}\}$ in Eq. (14), even with time-varying $\Delta_{i j}(t), \rho_{i}(t)$, and $\rho_{j}(t)$. The rest of the proof is identical to Theorem 2 .

If we select graph configuration A in Fig. 3, we have four symmetric phase differences available. We use two degrees of freedom as follows. We do not break symmetry of the right and left wings in the phase differences between the pitch and the lead-lag angles by setting

$$
\Delta_{76}=\Delta_{75}-\Delta_{65}=\Delta_{32}=\Delta_{31}-\Delta_{21}=-K_{\Delta_{32}} \theta_{b}+\Delta_{0}
$$

where $\theta_{b}$ denotes the pitch Euler angle, $K_{\Delta_{32}}$ is the control gain, and $\Delta_{0}$ is a nominal value that determines the steady-state pitch angle of the vehicle body $\left(\theta_{b}\right)$. This is effective in stabilizing the longitudinal motion, and the nominal value can be used to select ascent or decent angle.

To achieve perfect synchronization, we modify the coupled Hopf oscillators as discussed in Corollary 2. It is important to note that we do not have to differentiate $\Delta_{76}=\Delta_{32}$ to obtain $\dot{\mathbf{R}}\left(\Delta_{32}\right)$ in $\dot{\mathbf{T}}$. This follows from Eq. (44)

$$
\dot{\Delta}_{76}(t)=\dot{\Delta}_{32}(t)=K_{\Delta_{32}} \dot{\theta}_{b}=-K_{\Delta_{32}}\left(q \cos \phi_{b}-r \sin \phi_{b}\right)
$$

where we used the relationship between the body angular rate and the rate of the Euler angles given in Eq. (41).

To initiate a turning maneuver, consider breaking the symmetry of the right and left wings. One method of symmetry breaking is the maximum amplitude of the lead-lag angle ( $\rho_{3}$ and $\rho_{7}$ for the example in Fig. 3). We set this proportional to yaw rate to provide yaw-rate damping. Because of the coupled nature of the motions, this causes both the yaw rate and the roll angle to go to zero. If we want to control the bank angle directly by setting desired $\phi_{b}$, we can use

$$
\begin{aligned}
& \rho_{3}(t)=\rho_{3, \text { nominal }}-K_{r}\left(\phi_{b}-\phi_{b, \text { desired }}\right), \\
& \rho_{7}(t)=\rho_{7, \text { nominal }}+K_{r}\left(\phi_{b}-\phi_{b, \text { desired }}\right)
\end{aligned}
$$

where $\phi_{b, \text { desired }}$ can be set to zero to end turning. The coupled Hopf oscillators again can be modified as in Corollary 2 to achieve zero synchronization errors with the time-varying radii. We need not differentiate $\rho_{3}(t)$ and $\rho_{7}(t)$ directly, since

$$
\dot{\phi}_{b}=p+q \sin \phi_{b} \tan \theta_{b}+r \cos \phi_{b} \tan \theta_{b}
$$

We used the method of symmetry breaking for our simulation.

An alternative method of symmetry breaking is to add a difference value $\delta$ to the nominal value $(90 \mathrm{deg})$ of the phase differences of the flapping and pitch angles such that $\Delta_{65}=90 \mathrm{deg}+\delta$ and $\Delta_{21}=90 \mathrm{deg}-\delta$. The phase difference between flapping and pitch is vital for lift and thrust generation. Therefore, this difference between the right and left wings causes roll and proverse yaw. This method of symmetry breaking was observed in [66].

\section{Gliding Mode Control}

We produce gliding flight with no reciprocal flapping motion by setting the bifurcation parameter $\sigma=-1$ in Eq. (1). As discussed in Sec. II.D, simply setting $\omega(t)=0$ without $\sigma=-1$ will not ensure convergence to the controlled bias values $a_{i}$. This provides us with simple control of our wing by exploiting the bifurcation of Hopf oscillators, causing them to exponentially converge to a single nonoscillatory value corresponding to the bias $a_{i}$. We can then control the lead-lag motion $\psi_{w}$, flapping angle $\phi_{w}$, and wing pitch angle $\theta$ by their bias parameters. In gliding flight, synchronization is less critical, and so we can turn off the coupling by $k=0$. A negative (positive) flapping angle or negative (positive) lead-lag angle can provide a pitch-down (pitch-up) moment due to drag or lift, respectively. We have therefore reduced control dimensionality to three actively controlled parameters: wing pitch, wing flapping angle, and lead-lag angle. In fact, depending on the physical characteristics of the specific vehicle, controlling only one of wing flapping angle or lead-lag angle could be sufficient for longitudinal gliding stability. For the network in Fig. 3a, we can use the following bias for the lead-lag angle of each wing:

$$
a_{3}(t)=a_{7}(t)=-k_{p} \theta_{b}-k_{d} q-k_{i} \int_{0}^{t} \theta_{b} \mathrm{~d} t+\psi_{\text {bias }}
$$

where $k_{p}, k_{d}$, and $k_{i}$ are positive gains and $\psi_{\text {bias }}$ is a constant. The simulation uses proportional-integral-derivative control of flapping angle and lead-lag bias. The angle $\phi_{w}$, which implies a dihedral angle in gliding flight, turns out to be the most important, which is in keeping with a previous study of the importance of drag-based stability [72], and the conclusion that birds glide more like tailless 

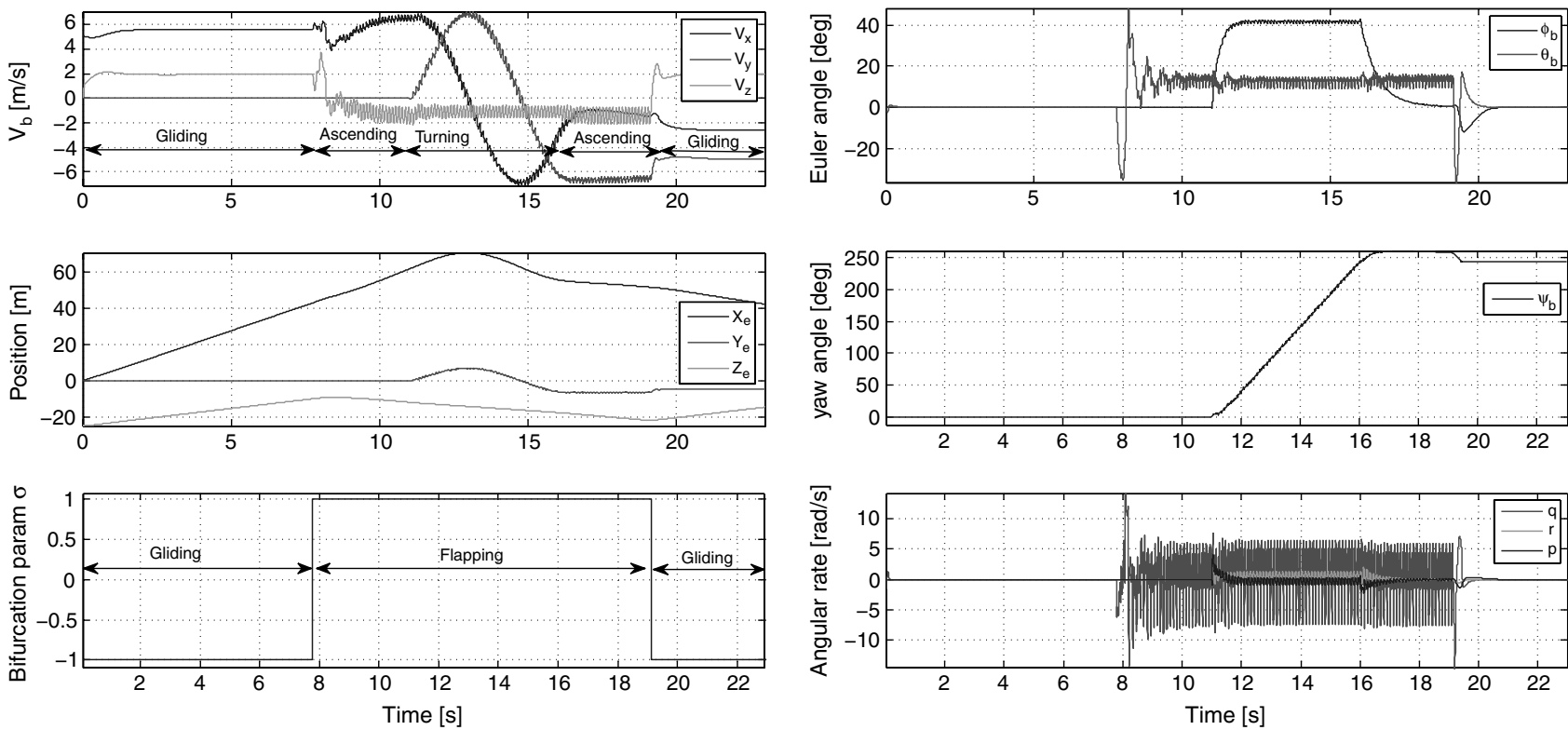

a) Body velocity and inertial position

b) Euler angles and angular rates

Fig. 7 State vectors of the two alternating flight modes, flapping, and gliding.
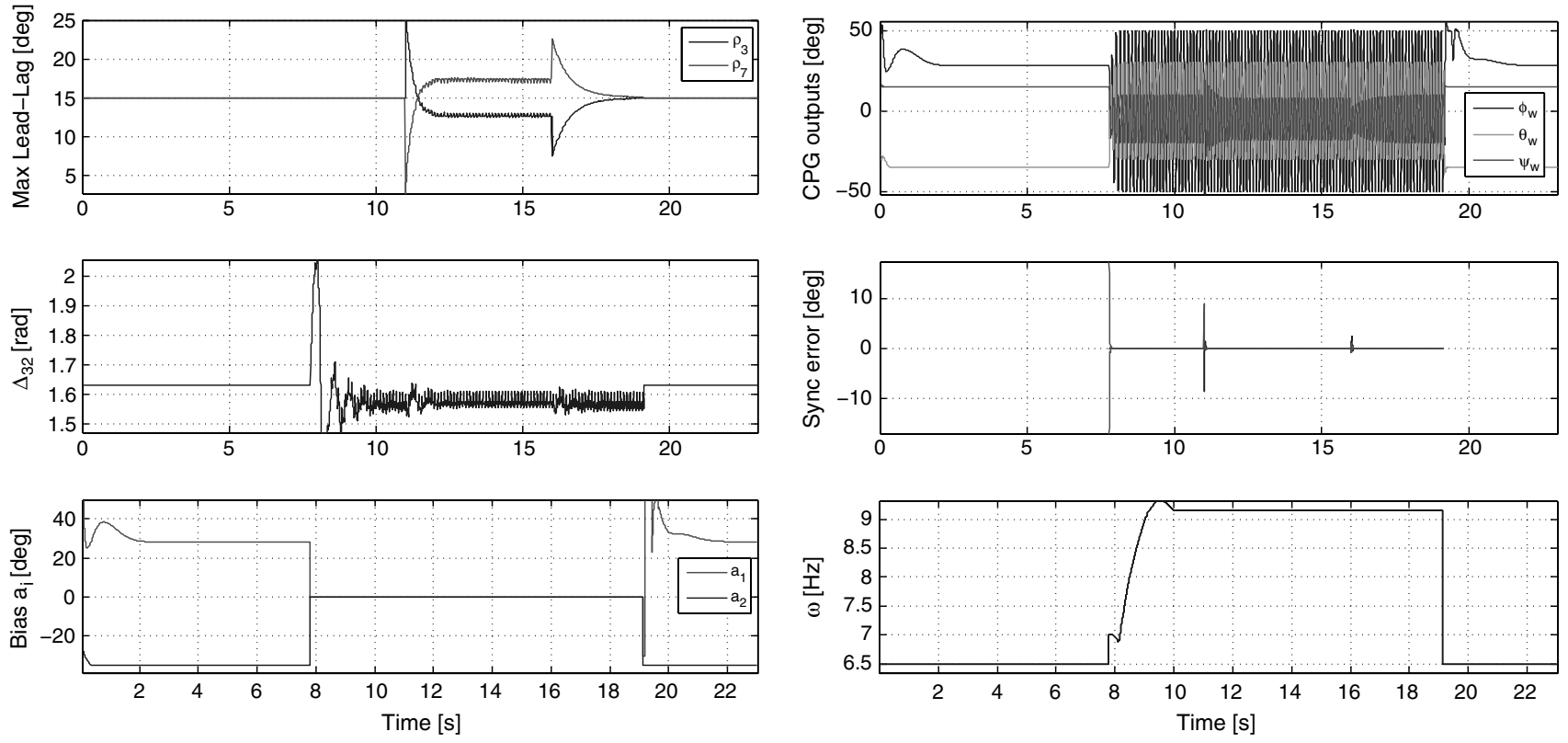

a) Control inputs

b) Wing joint angles, sync errors, and flapping frequency

Fig. 8 Control inputs and wing-joint angles of the two alternating flight modes, flapping and gliding.

vehicles than like conventional-tailed aircraft. Only integral control is used for pitch bias, to obtain a constant wing angle of attack.

\section{Two Alternating Flight Modes for Altitude Control}

Inspired by altitude stabilization of animal flight, we propose a switching logic between flapping mode and gliding mode. Requirements for switching use the current bifurcation parameter $\sigma \in$ $(-1,1)$ to determine what mode we are in, as well as altitude and velocity information to determine whether to switch mode. Recalling that the $z$ direction is positive downward, we set the test for gliding mode as

$$
\begin{array}{cc}
\text { if }\left(\sigma=1, \quad z_{b}<-h_{\text {max }, \text { flap }},\right. & \left.V_{b x}>V_{\mathrm{x}, \max }\right) \text { or } \\
\left(\sigma=-1, \quad z_{b}<-h_{\text {min, glide }},\right. & \left.V_{b x}>V_{\mathrm{x}, \min }\right)
\end{array}
$$

then glide $\sigma=-1$ by the control law in Sec. IV.A.3, else flap $\sigma=1$ by the control law in Secs. IV.A.1 and IV.A.2.

The switching logic ensures that we have sufficient altitude and forward velocity to glide but will interrupt the constant-ascending flapping flight with periods of gliding.

\section{B. Simulation Results}

We assume each wing to be a single rigid body. That is, in Eq. (26), we set $\left(x_{w}(r), y_{w}(r), z_{w}(r)\right)^{T}=0$. We also assume that the aerodynamic coefficients do not vary along the chord of the wing blade element. This dynamic model with three-dimensional wing kinematics is constructed in MATLAB/Simulink, allowing us to demonstrate how stability can be obtained for flapping flight driven by a CPG network (see Fig. 1). A simulation result of alternating flight modes by the control laws in Sec. IV.A is shown in Figs. 7 and 
Table 1 Key simulation parameters and nominal values

\begin{tabular}{lccc}
\hline \hline$m=0.3 \mathrm{~kg}$ & $\mathbf{I}_{b}=0.0012^{*}$ eye(3) $\mathrm{kg} \mathrm{m}^{2}$ & $R=0.32, c=0.15[\mathrm{~m}]$ & $k=60$ (flapping) or 0 (gliding) \\
$\Theta_{s}=20 \mathrm{deg}$ & $\lambda=10$ (flapping) & $\lambda=30$ (gliding) & $\rho_{1}=50, \rho_{2}=30, \rho_{3}=15[\mathrm{deg}]$ \\
$a_{1}=a_{5}=0$ & $a_{2}=a_{6}=0 \mathrm{deg}$ & $a_{3}=a_{7}=-5 \mathrm{deg}$ & Wing: $C_{L}, C_{D}:$ Eq. (30) \\
Wing: $C_{m 0}=-0.2$ & Wing: $C_{m \alpha}=-0.12$ & Body: $C_{m 0}=0.1$ & Body: $C_{m \alpha}=-0.2$ \\
\hline \hline
\end{tabular}

8. The control parameters and the wing angles driven by the CPG network are shown in Fig. 8. The key simulation parameters are listed in Table 1. As shown in Fig. 7, the vehicle begins in a gliding mode with $\sigma=-1$, transitions smoothly to ascending flapping flight $(\sigma=1)$ at $t=7.78 \mathrm{~s}$, and then executes a turn at $t=11 \mathrm{~s}$ while stabilizing both the longitudinal and the lateral modes. The vehicle resumes forward-ascending flapping flight by driving the bank angle to zero at $t=16 \mathrm{~s}$, and the ascending flapping flight switches to a glide mode as it reaches some desired maximum altitude $t=19.1 \mathrm{~s}$ (see $Z_{e}$ in Fig. 7a). Remarkably, the flapping-flying vehicle makes smooth transitions between various flight modes. This should be a testament to the potential of our proposed CPG-based control scheme.

To turn, at the $11 \mathrm{~s}$ mark, we set a desired bank angle $\phi_{b}=40 \mathrm{deg}$ for the symmetry-breaking control law in Eq. (46), accompanied by setting a constant frequency $(\dot{\omega}=0)$. What is interesting here is that the vehicle settles into a nice banked turn at the same flight-path angle without correcting the frequency of flapping. This is because the change of $\rho_{3}$ and $\rho_{7}$ causes the aerodynamic forces to increase in spite of a constant $\omega$. The bank angle, rate of turn, and qualitative characteristic (e.g., amplitude of body pitch oscillation) of the turn are interestingly linked to the scale and shift of the lead-lag coupling $\Delta_{32}$ and amplitude, but an exact correlation depends on physical and aerodynamical parameters. This must be further investigated and understood to implement a better nonlinear control law. The roll angle through the turn is about $40 \mathrm{deg}$ and the average global yaw rate is about $45 \mathrm{deg} / \mathrm{s}$, with oscillations between 5 and $75 \mathrm{deg} / \mathrm{s}$. This is feasible in light of Hedrick and Biewener's [66] experimentation. The local angle of attack of each wing varies along the wingspan $(r \in[0, R])$ and s nicely bounded between $\pm 50 \mathrm{deg}$ at the steadystate, as shown in Fig. 9. Although we have almost all positive angles of attack in the downstroke, creating high forces, the upstroke has a nice balance of positive and negative angles of attack.

Figure $8 \mathrm{~b}$ shows the resulting oscillatory behavior of the flapping $\phi_{w}$, pitch $\bar{\theta}_{w}$, and lead-lag motion $\psi_{w}$ commanded by the CPG network and highlights the effects of our changing control variables on CPG behavior. From arbitrary initial conditions, the CPG network synchronizes globally and exponentially. During gliding flight, the synchronization between the wing joints is less critical, so we set the
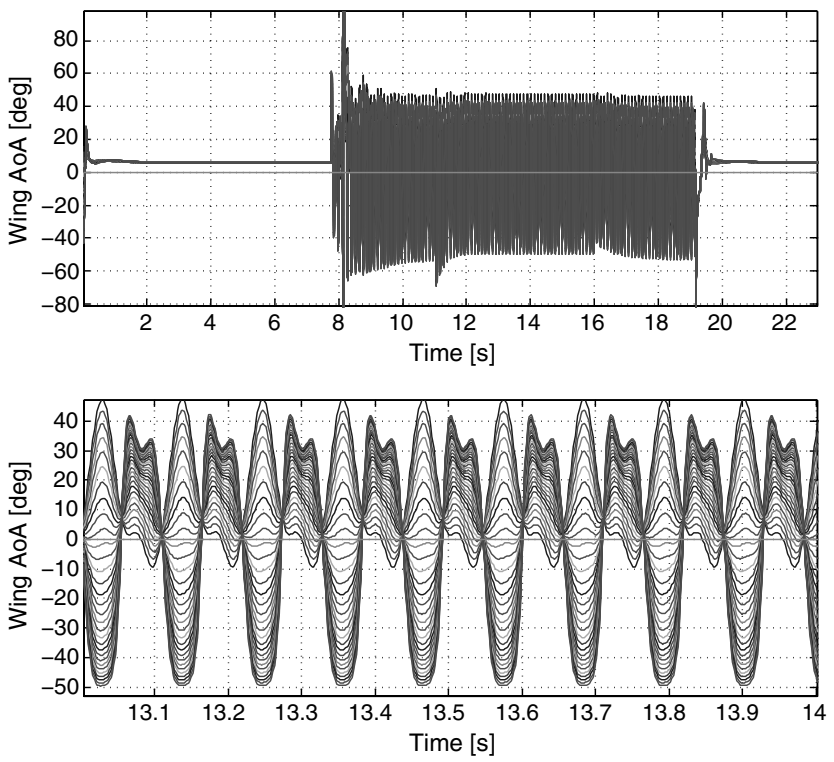

Fig. 9 Local angles of attack of the right wing along the wingspan $r \in[0, R] \mathrm{m}$ with $d r=0.01 \mathrm{~m}$. coupling gain $k=0$. Even if the phase difference $\Delta_{i j}$ and the amplitude oscillation $\rho_{i}$ are time-varying, the synchronization errors exponentially tend to zero, due to Corollary 2 and Theorem 2 . This simulation results supports our claim that our CPG-based flappingflight control can stabilize both the longitudinal and lateral dynamics by commanding three control parameters: $\omega(t), \Delta_{32}=\Delta_{76}$, and $\rho_{3} \neq \rho_{7}$.

\section{Conclusions}

We investigated the hypothesis that the phase control and synchronization of coupled nonlinear oscillators, inspired by central pattern generators (CPGs) found in animal spinal cords, can effectively produce and control stable flapping-flight patterns and can be used to stabilize the flapping-flying vehicle dynamics. An engineered CPG network, which ensures the stability and robust adaptation of motion, can significantly reduce the complexity associated with engineered flapping flight. Central to the agile flight of natural flyers is the ability to execute complex synchronized threedimensional motions of the wings. In this paper, we introduced a mathematical and control theoretic framework of CPG control theory that enabled such synchronized wing maneuvers. In contrast with an independent control of each wing joint by a sinusoidal function, the proposed CPG-based method used the phase differences between multiple wing oscillators as control mechanisms. We also showed that the central flight controller, similar to the brain of an animal, can stabilize the full 6-DOF vehicle dynamics by commanding a reduced number of control variables such as the frequency and phase difference of the oscillators instead of directly controlling multiple joints. Furthermore, to our knowledge, this paper presented the first result on alternating flight modes of gliding and flapping flight by using the Hopf bifurcation.

To show the effectiveness of the proposed CPG-based flappingflight control, we presented numerical simulation results by using a realistic vehicle dynamic model with three-dimensional wing kinematics. This dynamic model includes a tilted stroke-plane angle, the lead-lag motion, and the flapping and pitch angles of each wing, along with the full states of 6-DOF nonlinear vehicle dynamics. We also showed that CPG-based flight control can stabilize and alternate two different flight modes of flapping and gliding flight by using the synchronized and symmetry-breaking oscillatory motions of two main wings. This result is interesting in the sense that the tailless flapping-flight dynamics could be effectively controlled without using traditional aerodynamic control surfaces. This result agrees with a prior claim of biologists that birds act more like tailless aircraft.

Although we understand the challenges associated with lightweight low-power actuators to fully realize the potential of threedimensional wing movements, the research described in this paper can further enhance our understanding of key mechanisms of biological flyers. Ongoing work includes a study of aeroelasticity with various kinds of waveforms and compliant wings. It would also be important to identify which wing-joint variables can be controlled passively to further reduce the complexity.

\section{Acknowledgments}

This paper, which was awarded the AIAA Best Intelligent Systems Paper Award, was sponsored by the U.S. Air Force Office of Scientific Research under the Young Investigator Award Program (grant no. FA95500910089) monitored by W. Larkin. This paper benefited from discussion with J.-J. Slotine at Massachusetts Institute of Technology; K. Seo and K. Breuer at Brown University; and G. Abate, J. Evers, and D. Doman at the U.S. Air Force Research 
Laboratory. The robotic bat micro air vehicle was developed by the authors and the students at Iowa State University, including Jeremiah Stoner and Keegan Gartner.

\section{References}

[1] Mueller, T. J., Fixed and Flapping Wing Aerodynamics for Micro Air Vehicle Application, Progress in Astronautics and Aeronautics, AIAA, Reston, VA, 2001.

[2] Shyy, W., Lian, Y., Tang, J., Viieru, D., and Liu, H., Aerodynamics of Low Reynolds Number Flyers, Cambridge Univ. Press, New York, 2008.

[3] Azuma, A., The Biokinetics of Flying and Swimming, 2nd ed., AIAA, Reston, VA, 2006.

[4] Kato, N., and Kamimura, S., Bio-Mechanisms of Swimming and Flying: Fluid Dynamics, Biomimetic Robots, and Sports Science, SpringerVerlag, New York, 2008.

[5] Norberg, U.M., Vertebrate Flght: Mechanics, Physiology, Morphology, Ecology and Evolution, Springer-Verlag, New York, 1989.

[6] Chung, S.-J., and Slotine, J.-J. E., "Cooperative Robot Control and Concurrent Synchronization of Lagrangian Systems," IEEE Transactions on Robotics, Vol. 25, No. 3, 2009, pp. 686-700. doi:10.1109/TRO.2009.2014125

[7] Dickinson, W. B., and Dickinson, M. H., "The Effect of Advance Ratio on the Aerodynamics of Revolving Wings," Journal of Experimental Biology, Vol. 207, 2004, pp. 4269-4281. doi:10.1109/TRO.2009.201412510.1242/jeb.01266

[8] Ellington, C. P., "The Aerodynamics of Hovering Insect Flight. 1. The Quasi-Steady Analysis," Philosophical Transactions of the Royal Society of London, Series B: Biological Sciences, Vol. 305, No. 1122, 1984, pp. 1-15. doi:10.1098/rstb.1984.0049

[9] Ellington, C. P., "The Aerodynamics of Hovering Insect Flight. 2. Morphological Parameters," Philosophical Transactions of the Royal Society of London, Series B: Biological Sciences, Vol. 305, No. 1122, 1984, pp. $17-40$ doi:10.1098/rstb.1984.0050

[10] Ellington, C. P., "The Aerodynamics of Hovering Insect Flight. 3. Kinematics," Philosophical Transactions of the Royal Society of London, Series B: Biological Sciences, Vol. 305, No. 1122, 1984, pp. 41-78. doi:10.1098/rstb.1984.0051

[11] Ellington, C. P., "The Aerodynamics of Hovering Insect Flight. 4. Aerodynamic Mechanisms," Philosophical Transactions of the Royal Society of London, Series B: Biological Sciences, Vol. 305, No. 1122, 1984, pp. 79-113. doi:10.1098/rstb.1984.0052

[12] Ellington, C. P., "The Aerodynamics of Hovering Insect Flight. 5. A Vortex Theory," Philosophical Transactions of the Royal Society of London, Series B: Biological Sciences, Vol. 305, No. 1122, 1984, pp. 115-144. doi:10.1098/rstb. 1984.0053

[13] Ellington, C. P., "The Aerodynamics of Hovering Insect Flight. 6. Lift and Power Requirements," Philosophical Transactions of the Royal Society of London, Series B: Biological Sciences, Vol. 305, No. 1122, 1984, pp. 145-181. doi:10.1098/rstb.1984.0054

[14] Hall, K. C., Pigott, S. A., and Hall, S. R., "Power Requirements for Large-Amplitude Flapping Flight," Journal of Aircraft, Vol. 35, No. 3, 1998, pp. 352-361. doi: $10.2514 / 2.2324$

[15] Willis, D. J., Peraire, J., Drela, M., and White, J. K., "A Numerical Exploration of Parameter Dependence in Power Optimal Flapping Flight," AIAA Paper 2006-2994, 2006.

[16] Wang, Z. J., "Aerodynamic Efficiency of Flapping Flight: Analysis of a Two-Stroke Model," The Journal of Experimental Biology, Vol. 211, 2008, pp. 234-238. doi:10.1242/jeb.013797

[17] Roget, B., Sitaraman, J., Harmon, R., Grauer, J., Conroy, J., Hubbard, J., and Humbert, S., "A Computational Study of Flexible Wing Ornithopter Flight," 26th Applied Aerodynamics Conference, AIAA 2008-6397, Honolulu, HI, 2008

[18] Albertani, R. J., Stanford, B., DeLoach, R., Hubner, J. P., and Ifju, P., "Wind-tunnel Testing and Modeling of a Micro Air Vehicle with Flexible Wings," Journal of Aircraft, Vol. 45, No. 3, 2008, pp. 10251032 .

doi: $10.2514 / 1.33338$

[19] Ho, S., Nassef, H., Pornsinsirirak, N., Tai, Y.-C., and Ho, C.-M.,
"Unsteady Aerodynamics and Flow Control for Flapping Wing Flyers," Progress in Aerospace Sciences, Vol. 39, No. 8, 2003, pp. 635-681. doi:10.1016/j.paerosci.2003.04.001

[20] Dickinson, M. H., Lehmann, F. O., and Sane, S. P., "Wing Rotation and the Aerodynamic Basis of Insect Flight,” Science, Vol. 284, June 1999, pp. 1954-1960.

doi:10.1126/science.284.5422.1954

[21] Swartz, S. M., Iriarte-Diaz, J., Riskin, D. K., Song, A., Tian, X., Willis, D. J., and Breuer, K. S., "Wing Structure and the Aerodynamic Basis of Flight in Bats," 45th AIAA Aerospace Science Meeting, Reno, NV, AIAA Paper 2007-42, 2007.

[22] Tian, X., Iriarte-Diaz, J., Middleton, K., Galvao, R., Israeli, E., Roemer, A., Sullivan, A., Song, A., Swartz, S., and Breuer, K., "Direct Measurements of the Kinematics and Dynamics of Bat Flight," Bioinspiration \& Biomimetics, Vol. 1, No. 4, 2006, pp. S10-S19. doi:10.1088/1748-3182/1/4/S02

[23] Deng, X., Schenato, L., Wu, W. C., and Sastry, S. S., "Flapping Flight for Biomimetic Robotic Insects: Part 1-System Modeling," IEEE Transactions on Robotics, Vol. 22, No. 4, 2006, pp. 776-788. doi:10.1109/TRO.2006.875480

[24] Deng, X., Schenato, L., Wu, W. C., and Sastry, S. S., "Flapping Flight for Biomimetic Robotic Insects: Part 2-Flight Control Design," IEEE Transactions on Robotics, Vol. 22, No. 4, 2006, pp. 789-803. doi:10.1109/TRO.2006.875483

[25] Chung, S.-J., Dorothy, M., and Stoner, J. R., "Neurobiologically Inspired Control of Engineered Flapping Flight," AIAA Infotech at Aerospace and Unmanned Unlimited Conference and Exhibit, Seattle, WA, AIAA Paper 2009-1929, Apr. 2009.

[26] Oppenheimer, M., Doman, D., and Sigthorsson, D., "Dynamics and Control of a Minimally Actuated Biomimetic Vehicle: Part 2Control," AIAA Guidance, Navigation, and Control Conference, AIAA Paper 2009-6161, Chicago, Aug. 2009.

[27] Frampton, K. D., Goldfarb, M., Monopoli, D., and Cveticanin, D., "Passive Aeroelastic Tailoring for Optimal Flapping Wings," Fixed and Flapping Wing Aerodynamics for Micro Air Vehicle Application, edited by E. T. J. Mueller, AIAA, Reston, VA, 2001, pp. 473-482.

[28] Issac, K. K., and Agrawal, S. K., "An Investigation Into the Use of Springs and Wing Motions to Minimum the Power Expended by a Pigeon-Sized Mechanical Bird for Steady Flight," Journal of Mechanical Design, Vol. 129, No. 4, Apr. 2007, pp. 381-389. doi:10.1115/1.2429696

[29] Jones, K. D., Lund, T. C., and Platzer, M. F., "Experimental and Computational Investigation of Flapping Wing Propulsion," Fixed and Flapping Wing Aerodynamics for Micro Air Vehicle Application, edited by E. T. J. Mueller, AIAA, Reston, VA, 2001, pp. 307-336.

[30] Kawamura, Y., Souda, S., Nishimoto, S., and Ellington, C. P., "Clapping-Wing Micro Air Vehicle of Insect Size," Bio-Mechanisms of Swimming and Flying: Fluid Dynamics, Biomimetic Robots, and Sports Science, edited by N. Kato and S. Kamimura, Springer-Verlag, New York, 2008, pp. 319-330.

[31] Larijani, R. F., and DeLaurier, J. D., "A Nonlinear Aeroelastic Model for the Study of Flapping Wing Flight," Fixed and Flapping Wing Aerodynamics for Micro Air Vehicle Application, edited by E. T. J. Mueller, AIAA, Reston, VA, 2001, pp. 399-428.

[32] Michelson, R., Helmick, D., Reece, S., and Amarena, C., "A Reciprocating Chemical Muscle (RCM) for Micro Air Vehicle Entomopter Flight," Proceedings of the International Association for Unmanned Vehicle Systems, 1997, pp. 429-435.

[33] Steltz, E., Avadhanula, S., and Fearing, R. S., "High Lift Force with 275 $\mathrm{Hz}$ Wing Beat in MFI," IEEE International Conference on Intelligent Robots and Systems, Inst. of Electrical and Electronics Engineers, Piscataway, NJ, 2007, pp. 3987-3992.

[34] Wood, R. J., "The First Takeoff of a Biologically-Inspired At-Scale Robotic Insect," IEEE Transactions on Robotics, Vol. 24, No. 2, 2008, pp. 341-347. doi:10.1109/TRO.2008.916997

[35] Swartz, S. M., "Allometric Patterning in the Limb Skeleton of Bats: Implications for the Mechanics and Energetics of Powered Flight," Journal of Morphology, Vol. 234, No. 3, 1997, pp. 277-294. doi:10.1002/(SICI)1097-4687(199712)234:3<277::AID-JMOR6>3.0. $\mathrm{CO} ; 2-6$

[36] Swartz, S. M., Bishop, K. L., and Ismael-Aguirrre, M.-F., "Dynamic Complexity of Wing Form in Bats: Implications for Flight Performance," Functional and Evolutionary Ecology of Bats, Oxford Univ. Press, Oxford, UK, 2005.

[37] Taylor, G. K., and Zbikowski, R., "Nonlinear Time-periodic Models of the Longitudinal Flight Dynamics of Desert Locusts Schistocerca Gregaria," Journal of the Royal Society Interface, Vol. 2, No. 3, 2005, pp. 197-221. 
doi:10.1098/rsif.2005.0036

[38] Zbikowski, R., Ansari, S. A., and Knowles, K., "On Mathematical Modelling of Insect Flight Dynamics in the Context of Micro Air Vehicles," Bioinspiration \& Biomimetics, Vol. 1, No. 2, 2006, pp. 26-37. doi:10.1088/1748-3182/1/2/R02

[39] Hooper, S. L., "Central Pattern Generators," Nature Encyclopedia of Life Sciences, Wiley, New York, 2001.

[40] Bay, J. S., and Hemami, H., "Modeling of a Neural Pattern Generator with Coupled Nonlinear Oscillators," IEEE Transactions on Biomedical Engineering, Vol. 34, No. 4, pp. 297-306. doi:10.1109/TBME.1987.326091, 1987.

[41] Bernstein, N., The Coordination and Regulation of Movements, Pergamon, Oxford, 1967.

[42] Collins, J. J., and Stewart, I. N., "Coupled Nonlinear Oscillators and the Symmetries of Animal Gaits," Nonlinear Science Today, Vol. 3, No. 1, 1993, pp. 349-392. doi:10.1007/BF02429870

[43] Golubitsky, M., Stewart, I., Buono, P., and Collins, J., "Symmetry in Locomotor Central Pattern Generators and Animal Gaits," Nature, Vol. 401, No. 6754, 1999, pp. 693-695. doi: $10.1038 / 44416$

[44] Hooper, S. L., Guschlbauer, C., von Uckermann, G., and Buschges, A., "Natural Neural Output That Produces Highly Variable Locomotory Movements," Journal of Neurophysiology, Vol. 96, No. 4, 2006, pp. 2072-2088. doi:10.1152/jn.00366.2006

[45] Lewis, M. A., Tenore, F., and Etienne-Cummings, R., "CPG Design Using Inhibitory Networks," Proceedings of the 2005 IEEE International Conference on Robotics and Automation, Inst. of Electrical and Electronics Engineers, Piscataway, NJ, 2005, pp. 36823687.

[46] Matsuoka, K., "Mechanisms of Frequency and Pattern Control in Neural Rhythm Generators," Biological Cybernetics, Vol. 56, Nos. 5-6, 1987, pp. 345-353. doi:10.1007/BF00319514

[47] Sekerli, M., and Butera, R. J., "Oscillations in a Simple Neuromechanical System: Underlying Mechanisms," Journal of Computational Neuroscience, Vol. 19, No. 2, 2005, pp. 181-197. doi:10.1007/s10827-005-1537-y

[48] Grillner, S., "Neurological Bases of Rhythmic Motor Acts in Vetebrates," Science, Vol. 228, No. 4696, 1985, pp. 143-149. doi:10.1126/science.3975635

[49] Iwasaki, T., and Zheng, M., "Sensory Feedback Mechanism Underlying Entrainment of Central Pattern Generator to Mechanical Resonance," Biological Cybernetics, Vol. 94, No. 4, 2006, pp. 245-261. doi:10.1007/s00422-005-0047-3

[50] Seo, K., Chung, S.-J., and Slotine, J.-J. E., "CPG-Based Control of a Turtle-Like Underwater Vehicle," Proceedings of the Robotics, Science and Systems (RSS), June 2008.

[51] Seo, K., Chung, S.-J., and Slotine, J.-J. E., "CPG-based Control of a Turtle-Like Underwater Vehicle," Autonomous Robots (to be published).

[52] Ijspeert, A. J., "Central Pattern Generators for Locomotion Control in Animals and Robots: A Review," Neural Networks, Vol. 21, No. 4, 2008, pp. 642-653. doi:10.1016/j.neunet.2008.03.014

[53] Ayers, J., and Rulkov, N., "Controlling Biomimetic Underwater Robots with Electronic Nervous Systems," Bio-Mechanisms of Swimming and Flying: Fluid Dynamics, Biomimetic Robots, and Sports Science, edited by N. Kato, and E. S. Kamimura, Springer-Verlag, New York, 2008, pp. 295-306.

[54] Ijspeert, A. J., Crespi, A., and Cabelguen, J.-M., "Simulation and Robotics Studies of Salamander Locomotion: Applying Neurobiological Principles to the Control of Locomotion in Robots," Neuroinformatics, Vol. 3, No. 3, 2005, pp. 171-195. doi:10.1385/NI:3:3:171

[55] Morimoto, J., Endo, G., Nakanishi, J., and Cheng, G., "A Biologically Inspired Biped Locomotion Strategy for Humanoid Robots:
Modulation of Sinusoidal Patterns by a Coupled Oscillator Model,' IEEE Transactions on Robotics, Vol. 24, No. 1, pp. 185-191. doi:10.1109/TRO.2008.915457, 2008.

[56] Strogatz, S., Nonlinear Dynamics and Chaos With Applications to Physics, Biology, Chemistry, and Engineering, Perseus Books Group, Cambridge, MA, 1994.

[57] Slotine, J.-J. E., and Li, W., Applied Nonlinear Control, Prentice-Hall, Upper Saddle River, NJ, 1991.

[58] Ijspeert, A. J., Crespi, A., Ryczko, D., and Cabelguen, J.-M., "From Swimming to Walking with a Salamander Robot Driven by a Spinal Cord Model," Science, Vol. 315, No. 5817, 2007, pp. 1416-1420. doi:10.1126/science. 1138353

[59] Liberzon, D., Switching in Systems and Control, Birkhäuser Boston, 2003.

[60] Pham, Q.-C., and Slotine, J.-J. E., "Stable Concurrent Synchronization in Dynamic System Networks," Neural Networks, Vol. 20, No. 1, 2007, pp. 62-77. doi:10.1016/i.neunet.2006.07.008

[61] Flash, T., and Hochner, B., "Motor Primitives in Vertebrates and Invertebrates," Current Opinion in Neurobiology, Vol. 15, No. 6, 2005, pp. 660-666. doi:10.1016/i.conb.2005.10.011

[62] Doya, K., Kimura, H., and Kawato, M., "Neural Mechanisms of Learning and Control," IEEE Control Systems Magazine, Vol. 21, No. 4, 2001, pp. 42-54. doi:10.1109/37.939943

[63] Norberg, U. M., and Winter, Y., "Wing Beat Kinematics of a NectarFeeding Bat, Glossophaga Soricina, Flying at Different Flight Speeds and Strouhal Numbers," Journal of Experimental Biology, Vol. 209, No. 19,2006 , pp. 3887-3897. doi: $10.1242 /$ jeb.02446

[64] Taga, G., "A Model of the Neuro-Musculo-Skeletal System for Anticipatory Adjustment of Human Locomotion During Obstacle Avoidance," Biological Cybernetics, Vol. 78, No. 1, 1998, pp. 9-17. doi: $10.1007 / \mathrm{s} 004220050408$

[65] Riskin, D. K., Willis, D. J., Iriarte-Diaz, J., Hendrick, T. L., Kostandov, M., Chen, J., Laidlaw, D. H., Breuer, K. S., and Swartz, S. M., "Quantifying the Complexity of Bat Wing Kinematics," Journal of Theoretical Biology, Vol. 254, No. 3, 2008, pp. 604-615. doi:10.1016/j.jtbi.2008.06.011

[66] Hedrick, T. L., and Biewener, A. A., "Experimental Study of Low Speed Turning Flight in Cockatoos and Cockatiels," 45th AIAA Aerospace Sciences Meeting and Exhibit, Reno, NV, AIAA Paper 2007-0044, Jan. 2007.

[67] Lohmiller, W., and Slotine, J.-J.E., "Contraction Analysis for Nonlinear Systems," Automatica, Vol. 34, No. 6, 1998, pp. 683-696. doi:10.1016/S0005-1098(98)00019-3

[68] Iwasaki, T., and Zheng, M., "What Makes Biological Oscillators Achieve Robust Self-Excitation?," Proceedings of the American Control Conference, Inst. of Electrical and Electronics Engineers, Piscataway, NJ, June 2003, pp. 1824-1829.

[69] Taga, G., Yamaguchi, Y., and Shimizu, H., "Self-organized Control of Bipedal Locomotion by Neural Oscillators in Unpredictable Environment," Biological Cybernetics, Vol. 65, No. 3, 1991, pp. 147-159. doi:10.1007/BF00198086

[70] Morimoto, J., Endo, G., Nakanishi, J., Hyon, S.-H., Cheng, G., Bentivegna, D., and Atkeson, C. G., "Modulation of Simple Sinusoidal Patterns by a Coupled Oscillator Model for Biped Walking," Proceedings of the 2006 IEEE International Conference on Robotics and Automation, Inst. of Electrical and Electronics Engineers, Piscataway, NJ, 2006, pp. 1579-1584.

[71] MacMahon, T. A., Muscles, Reflexes, and Locomotion, Princeton Univ. Press, Princeton, NJ, 1984.

[72] Thomas, A. L. R., and Taylor, G. K., "Animal Flight Dynamics I. Stability in Gliding Flight," Journal of Theoretical Biology, Vol. 212, No. 3, 2001, pp. 399-424. doi: $10.1006 /$ jtbi.2001.2387 